\title{
Shigaraki UAV-Radar Experiment (ShUREX): overview of the campaign with some preliminary results
}

\author{
Lakshmi Kantha ${ }^{1}$, Dale Lawrence ${ }^{1}$, Hubert Luce ${ }^{2}$, Hiroyuki Hashiguchi ${ }^{3 *}$, Toshitaka Tsuda ${ }^{3}$, Richard Wilson ${ }^{4}$,
} Tyler Mixa ${ }^{1}$ and Masanori Yabuki ${ }^{3}$

\begin{abstract}
The Shigaraki unmanned aerial vehicle (UAV)-Radar Experiment (ShUREX) is an international (USA-JapanFrance) observational campaign, whose overarching goal is to demonstrate the utility of small, lightweight, inexpensive, autonomous UAVs in probing and monitoring the lower troposphere and to promote synergistic use of UAVs and very high frequency (VHF) radars. The 2-week campaign lasting from June 1 to June 14, 2015, was carried out at the Middle and Upper Atmosphere (MU) Observatory in Shigaraki, Japan. During the campaign, the DataHawk UAV, developed at the University of Colorado, Boulder, and equipped with high-frequency response cold wire and pitot tube sensors (as well as an iMET radiosonde), was flown near and over the VHF-band MU radar. Measurements in the atmospheric column in the immediate vicinity of the radar were obtained. Simultaneous and continuous operation of the radar in range imaging mode enabled fine-scale structures in the atmosphere to be visualized by the radar. It also permitted the UAV to be commanded to sample interesting structures, guided in near real time by the radar images. This overview provides a description of the ShUREX campaign and some interesting but preliminary results of the very first simultaneous and intensive probing of turbulent structures by UAVs and the MU radar. The campaign demonstrated the validity and utility of the radar range imaging technique in obtaining very high vertical resolution ( $20 \mathrm{~m})$ images of echo power in the atmospheric column, which display evolving fine-scale atmospheric structures in unprecedented detail. The campaign also permitted for the very first time the evaluation of the consistency of turbulent kinetic energy dissipation rates in turbulent structures inferred from the spectral broadening of the backscattered radar signal and direct, in situ measurements by the highfrequency response velocity sensor on the UAV. The data also enabled other turbulence parameters such as the temperature structure function parameter $C_{T}^{2}$ and refractive index structure function parameter $C_{n}^{2}$ to be measured by sensors on the UAV, along with radar-inferred refractive index structure function parameter $C_{n \text {,radar. }}^{2}$. The comprehensive dataset collected during the campaign (from the radar, the UAV, the boundary layer lidar, the ceilometer, and radiosondes) is expected to help obtain a better understanding of turbulent atmospheric structures, as well as arrive at a better interpretation of the radar data.
\end{abstract}

Keywords: VHF radar, MU radar, MST radar, Unmanned aerial vehicles, UAV, Turbulent mixing, Sheets and layers, High humidity gradient sheets, Mid-level cloud-base turbulence, Convective boundary layer, Kelvin-Helmholtz instability, Troposphere, Moist troposphere

\footnotetext{
*Correspondence: hasiguti@rish.kyoto-u.ac.jp

${ }^{3}$ Research Institute for Sustainable Humanosphere, Kyoto University, Kyoto,

Japan

Full list of author information is available at the end of the article
} 


\section{Introduction}

The overarching goal of the Shigaraki unmanned aerial vehicle (UAV)-Radar Experiment (ShUREX) campaign is to demonstrate the utility of small, light weight, inexpensive, autonomous UAVs in probing and monitoring the lower atmosphere in a controlled manner. An auxiliary goal is to demonstrate the synergistic use of UAVs and very high frequency (VHF) stratosphere-troposphere (ST) radars in measuring properties in structures of interest in the atmospheric column. This includes turbulence in the atmospheric boundary layer $(\mathrm{ABL})$, turbulence aloft created by mid-level, cloud-base convection (MCT) and Kelvin-Helmholtz instability (KHI), and "sheets and layers" (SL) structures in stably stratified conditions. More specifically, the campaign aims

1. To obtain in situ measurements of the turbulence kinetic energy (TKE) dissipation rate $\varepsilon$ from the UAV-borne fast response velocity sensor, along with simultaneous measurements by the Middle and Upper Atmosphere (MU) radar from spectral broadening of the backscattered radio signals

2. To obtain in situ measurements of the temperature structure function parameter $C_{T}^{2}$ from the UAV-borne fast response temperature sensor

3. To obtain in situ measurements of the refractive index structure function parameter $C_{n}^{2}$ in the atmospheric column from UAV-borne temperature and humidity sensors, along with simultaneous measurements of $C_{n}^{2}$ by the MU radar

To achieve these goals, the DataHawk UAV, developed at University of Colorado, was equipped with fast response $(100 \mathrm{~Hz})$ cold wire $(\mathrm{CW})$ temperature and velocity (pitot tube) sensors. The MU radar was operated simultaneously in range imaging mode to obtain high vertical resolution. The details of their operation are provided below.

Atmospheric measurements by small, low-cost UAVs have become increasingly popular in recent years (e.g., van den Kroonenberg et al. 2008; Lawrence et al. 2008; Balsley et al. 2013; Bonin et al. 2015; Scipion et al. 2016), because they offer many potential advantages for atmospheric studies (e.g., Lawrence and Balsley 2013). Scipion et al. (2016) have also used the DataHawk for simultaneous measurements and comparisons of $C_{n}^{2}$ with SOUSY (SOUnding SYstem) radar in Jicamarca, Peru.

\section{Methods/Experimental \\ Overview of measuring instruments MU radar}

The MU radar is a pulsed VHF Doppler radar located at the Shigaraki MU Observatory $\left(34.854061^{\circ} \mathrm{N}, 136.105606^{\circ} \mathrm{E}\right)$, Japan, $378 \mathrm{~m}$ above sea level (ASL) (Fig. 1). It is operated by

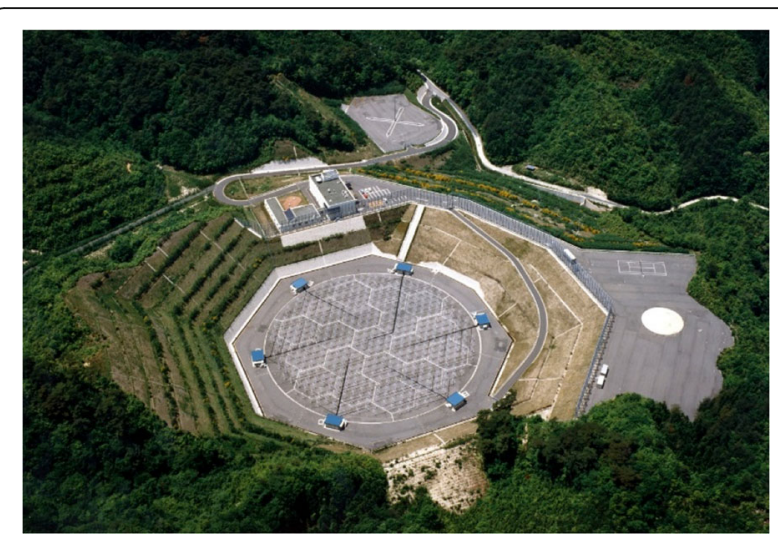

Fig. 1 The MU radar at Shigaraki MU Observatory, Japan (see the text for technical specifications)

the Research Institute for Sustainable Humanosphere (RISH) of the Kyoto University (see Fukao et al. 1990). In standard mode, the radar operates at $46.5 \mathrm{MHz}$ with a bandwidth of $3.5 \mathrm{MHz}$ and a peak output power of $1 \mathrm{MW}$ and consists of a $103 \mathrm{~m}$ diameter array of 475 Yagi antennas that can be steered electronically. During the campaign, the radar was operated in a range imaging mode using frequency diversity at vertical incidence (e.g., Palmer et al. 1999; Luce et al. 2001). The details of the radar parameters are given in Table 1. Owing to the radar parameter configuration used, high altitude resolution of a few tens of meters (typically $\sim 20 \mathrm{~m}$ ) can be achieved for high signal to noise ratios, using the Capon processing method. This method has been used

Table 1 MU radar parameters used during the ShUREX 2015 campaign

\begin{tabular}{ll}
\hline Parameter & \\
\hline Beam directions & $\left(0^{\circ}, 0^{\circ}\right),\left(0^{\circ}, 0^{\circ}\right),\left(0^{\circ}, 0^{\circ}\right),\left(0^{\circ}, 0^{\circ}\right),\left(0^{\circ}, 0^{\circ}\right)$, \\
& $\left(0^{\circ}, 10^{\circ}\right),\left(45^{\circ}, 10^{\circ}\right),\left(90^{\circ}, 10^{\circ}\right),\left(135^{\circ}, 10^{\circ}\right),\left(180^{\circ}, 10^{\circ}\right)$ \\
Radar frequencies (MHz) & $46.00,46.25,46.50,46.75,47.00$ \\
Interpulse period ( $\mu \mathrm{s})$ & 400 \\
Subpulse duration ( $\mu \mathrm{s})$ & 1 \\
Pulse coding & 16 -bit optimal code \\
Range resolution (m) & $150 \mathrm{~m}$ (nominal), 20 m (range imaging) \\
Height sampling (FII) (m) & 5 \\
Number of gates & 128 \\
Coherent integration & 32 \\
number & \\
Incoherent integration & $-($ time series) \\
number & \\
Number of FFT points & 128 \\
Acquisition time (s) & $16.384^{\mathrm{a}}$ \\
Time sampling (s) & 4.096 \\
Nyquist frequency (Hz) & 3.9063 \\
Velocity aliasing (ms ${ }^{-1}$ ) & 12.6 \\
\hline
\end{tabular}

${ }^{a}$ Acquisition of a time series of 128 points for each direction but the effective time resolution is about half $(\sim 8 \mathrm{~s})$ due to Hanning windowing of the time series 
with the MU radar on many occasions (e.g., Luce et al. 2007) and has been demonstrated to be effective for resolving thin and horizontally stratified temperature and humidity gradients, as well as for delineating deep turbulent regions (e.g., Luce et al. 2014). Despite this, the method is still not fully recognized and accepted by the ST radar community (e.g., Hocking 2011). As an aside of the main objectives of the campaign, a dataset from a specially designed UAV flight around the radar was used to validate the Capon method for retrieving HR images of echo power and hence reinforce the capability of the radar to resolve fine-scale structures in the atmosphere, as shown in the section "Detection of UAVs by MU Radar."

The Shigaraki MU Observatory also houses a UHF wind profiler, a boundary layer lidar, a Rayleigh Raman lidar, and a ceilometer. The facility also enables launch of radiosondes and ozonesondes at the site.

Since its establishment in 1984, the MU radar has contributed significantly to our knowledge of turbulence, and gravity waves and their role in middle atmosphere dynamics (e.g., Fukao and Hamazu 2014, see also reviews by Kato 2005; Tsuda 2014). Under some important, but not necessarily robust hypotheses, turbulence parameters can be retrieved, mainly from the radar echo power and from the spectral width of Doppler echoes (e.g., Hocking 1985, 1999; Fukao et al. 1994). However, there are still some pending issues about the accuracy (and validity) of the VHF radar-derived turbulence parameter estimates, because the data processing methods used are difficult to validate and because the VHF radar backscatter mechanisms are complex and still not fully understood (e.g., Röttger and Larsen 1990; Hocking 2011). Quantitative comparisons with estimates from simultaneous in situ radiosonde (balloon) measurements are useful for better understanding the radar observations (e.g., Luce et al. 2014) but difficult due to the different nature of the two measurements. The radar measurements provide volume- and timeaveraged, remotely sensed parameters at a fixed location, while balloon measurements are local and nearly instantaneous along the balloon path, but semi-Lagrangian. A rigorous comparison of balloon measurements with radar measurements is problematic because the radiosondes drift away, often quite rapidly, from the radar location due to horizontal winds. The horizontal inhomogeneity and intermittency inherent to turbulence can therefore prevent meaningful comparisons from being made.

On the other hand, a UAV equipped with appropriate sensors and flown over or close to the radar enables meaningful comparisons to be made. Indeed, in situ turbulence parameter measurements over and/or in the immediate proximity of the radar can help interpret radar measurements and vice versa. UAVs also enable probing of important turbulent structures in the atmospheric column in real or near real time, guided by radar signals.

\section{DataHawk UAV}

Small (less than $2 \mathrm{~m}$ wingspan), light weight (about $1 \mathrm{~kg}$ mass), inexpensive ( $\$ 1000$ to $\$ 2000)$, autonomous DataHawk UAVs have been developed at the University of Colorado. They are equipped with autopilots and can be pre-programmed to execute a preplanned trajectory so that the sensors on board can make atmospheric measurements along that preplanned trajectory. This trajectory can be a spiraling ascent to a prescribed altitude over a particular spot and descent but can also be a racetrack/ladder-type trajectory. High-resolution velocity and temperature sensors, capable of yielding information on turbulence, in addition to standard PTU (i.e., pressure, temperature and relative humidity) measurements, can be deployed on the UAVs. The UAVs can be catapult (bungee-cord) launched or taken aloft by a standard weather balloon and deployed from a height to conserve battery power and prolong measurements. Balloon launches save battery power and enable longer flights, as long as such launches are permitted by the aviation authorities. However, there are restrictions. Often, the authorities impose a ceiling on UAV flights to prevent interference with commercial aircraft, when the launch site is under the flight path of nearby busy airports.

A picture of a DataHawk UAV is shown in Fig. 2. The wingspan of the current version is $1.5 \mathrm{~m}$ and its mass is $1.1 \mathrm{~kg}$. It has also the unique capability to make relative wind and temperature measurements with fast response $(100 \mathrm{~Hz})$ pitot tube and cold wire $(\mathrm{CW})$ sensors, respectively, for estimating dynamic and temperature turbulence parameters. It is propelled by a pusher prop in the rear, powered by an electric motor running on a $11 \mathrm{~V}, 7.6 \mathrm{Ah}$ LiPo battery pack. It can be bungee- or balloon-launched and has an endurance of $\sim 60$ to 70 min depending on the climb rate. The DataHawk UAV airspeed ranges from 10 to $25 \mathrm{~m} \mathrm{~s}^{-1}$. Depending on a variety of factors such as the flight control strategy and motor power, the UAV cannot fly in wind speeds larger than $10-15 \mathrm{~m} \mathrm{~s}^{-1}$, because of the risk of UAV loss. The range is around 20 to $40 \mathrm{~km}$, determined by the uplink/downlink antenna gain and the available line of sight. DataHawk uses global positioning system (GPS) for navigation. It has a custom-designed autopilot, which keeps the UAV on its preplanned trajectory from launch to recovery, and operators need only to supervise the flight (changing trajectory parameters when needed) but need not to fly the aircraft itself. However, the UAV can also be commanded by the operator in real time to sample a nearby atmospheric structure as indicated by, say, the radar images collected in near real time.

DataHawk UAV also carried pressure, humidity, and an additional slower response (but precalibrated) temperature sensor. These are designated as DataHawk (DH) sensors (Table 2), whose inputs, along with GPS and the pitot tube data, were used for UAV operation and flight control. For 


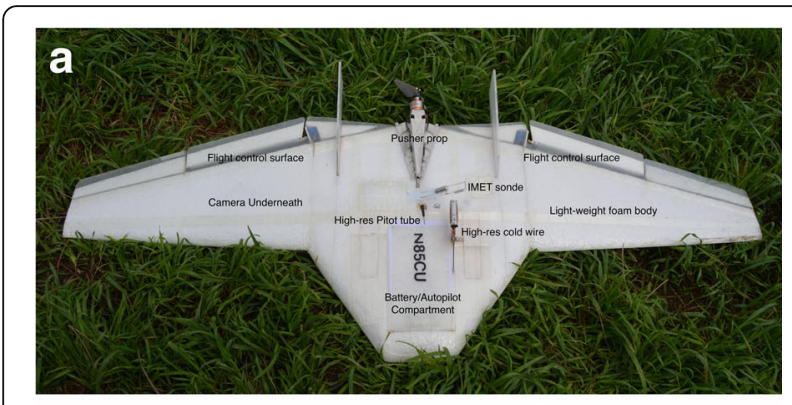

b

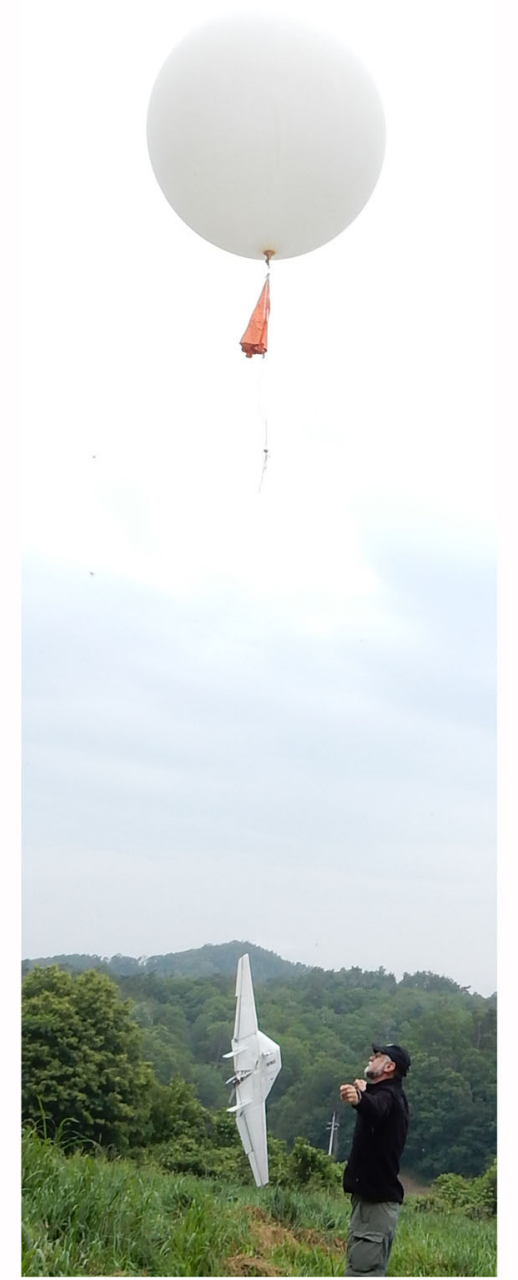

Fig. 2 a The University of Colorado DataHawk UAV. b Balloon launch

potential but future use in validating the Thorpe technique for estimating $\varepsilon$ from radiosondes (not a topic for this paper), a conventional radiosonde developed by International Met Systems (iMET) was also mounted on the UAV. Thus, the UAV carried three temperature sensors, two humidity sensors, two pressure sensors, and one velocity sensor, along with the UAV and iMET GPS sensors, providing multiple redundancy. Co-located pressure and GPS sensors enabled a more accurate determination of the UAV altitude, by combining the strengths but avoiding the deficiencies (such as GPS dropouts) of the two techniques. See Table 2 for sensor characteristics.

The DataHawk UAV carries a custom sensor module that provides high-resolution temperatures via a $5 \mu \mathrm{m}$ diameter platinum resistance temperature detector (RTD) wire. The wire is excited at constant current as a "coldwire," i.e., at a very low overheat ratio, so that its voltage drop is proportional to resistance, and therefore insensitive to the flow velocity, resulting in a temperature change less than $0.002{ }^{\circ} \mathrm{C} / \mathrm{ms}^{-1}$. These coldwire temperature measurements have a range of -60 to $+40{ }^{\circ} \mathrm{C}$, quantized at 16 bit resolution, for a digital temperature resolution of $0.0015{ }^{\circ} \mathrm{C}$. The coldwire itself has a thermal time constant of $0.6 \mathrm{~ms}$ at nominal $14 \mathrm{~ms}^{-1}$ airspeed. The voltage data is anti-aliased at $130 \mathrm{~Hz}$ and sampled at $700 \mathrm{~Hz}$, and subsequently subsampled at $100 \mathrm{~Hz}$ to remain with in the passband of the anti-alias filter. The coldwire is calibrated from voltage to degrees Celsius in post-flight analysis using a co-located commercial sensor (ADS1118) that has much slower frequency response than the coldwire (time constant about $2 \mathrm{~s}$ at $14 \mathrm{~ms}^{-1}$ airspeed) but has a specified accuracy better than $\pm 1{ }^{\circ} \mathrm{C}$ over -40 to $+125^{\circ} \mathrm{C}$ range (Table 2 ).

The DataHawk sensor module also contains a commercial humidity sensor (Honeywell HIH4031) that is relatively slow (time constant about $5 \mathrm{~s}$ at nominal flow rates). The factory calibration using temperature compensation is used to produce relative humidity $(\mathrm{RH})$ that is specified to $\pm 8 \%$ over the $0-100 \% \mathrm{RH}$ range and the -40 to $+85{ }^{\circ} \mathrm{C}$ temperature range (Table 2). The $\mathrm{RH}$ signal is sampled at $10 \mathrm{~Hz}$.

Velocity of the flow relative to the DataHawk is measured by a Pitot-static tube and differential pressure sensor (MS4525DO DS5AI001DP), mounted at a height of $3 \mathrm{~cm}$ above the vehicle to project into the free stream above the aerodynamic boundary layer. This sensor is sampled at $800 \mathrm{~Hz}$, but is subsampled at $100 \mathrm{~Hz}$ for the analysis to be consistent with the cold wire data. At the nominal airspeed of $14 \mathrm{~ms}^{-1}$, this measurement has a digital resolution of $0.042 \mathrm{~ms}^{-1}$.

The DataHawk UAV is well suited to making atmospheric measurements up to altitudes of $4.0 \mathrm{~km}$ (bungee launch) and $7.0 \mathrm{~km}$ (balloon launch). The design of the DataHawk UAV, the characteristics of ground support components, and some preliminary data collected have been described by Lawrence and Balsley (2013) and Balsley et al. (2013).

\section{Vaisala radiosondes}

Seven Vaisala radiosondes were launched during the campaign to complement UAV data, and the launches were coordinated with UAV launches. The goal was also to provide a good idea of the atmospheric column conditions above the maximum altitude of the UAV flight to facilitate interpretation of radar images. This dataset will not be analyzed in detail here. Analyses of the balloon dataset, including comparisons with radar measurements, will be 
Table 2 List of sensors on board DataHawk UAV

\begin{tabular}{llllll}
\hline Sensor type & Resolution & Accuracy & Range & Time constant & Update frequency \\
\hline DH GPS & $10 \mathrm{~cm}$ & $10 \mathrm{~m}$ & Global & $1 \mathrm{~s}$ & $5 \mathrm{~Hz}$ \\
DH pressure & $0.012 \mathrm{hPa}$ & $2.5 \mathrm{hPa}$ & 10 to $1200 \mathrm{hPa}$ & $8.22 \mathrm{~ms}$ & $44.4 \mathrm{~Hz}$ \\
DH temperature & $0.1{ }^{\circ} \mathrm{C}$ & $2{ }^{\circ} \mathrm{C}$ & -60 to $+40{ }^{\circ} \mathrm{C}$ & $5 \mathrm{~s}$ & $10 \mathrm{~Hz}$ \\
DH humidity & $0.01 \%$ & $2 \%$ & 0 to $100 \%$ & $5 \mathrm{~s}$ & $10 \mathrm{~Hz}$ \\
$\mathrm{CW}$ temperature & $0.0015^{\circ} \mathrm{C}$ & $2{ }^{\circ} \mathrm{C}$ & -60 to $+40{ }^{\circ} \mathrm{C}$ & $0.5 \mathrm{~ms}$ & $100 \mathrm{~Hz}$ \\
Pitot-static tube & $0.01 \mathrm{~ms}^{-1}$ & $0.2 \mathrm{~ms}^{-1}$ & 0 to $30 \mathrm{~ms}^{-1}$ & $0.3 \mathrm{~ms}$ & $100 \mathrm{~Hz}$ \\
iMET pressure & $0.01 \mathrm{hPa}^{\text {iMET temperature }}$ & $0.5 \mathrm{hPa}^{\circ}$ & 2 to $1070 \mathrm{hPa}$ & $1 \mathrm{~s}$ & $1 \mathrm{~Hz}$ \\
iMET humidity & $0.01{ }^{\circ} \mathrm{C}$ & $0.2{ }^{\circ} \mathrm{C}$ & -95 to $+50{ }^{\circ} \mathrm{C}$ & $2 \mathrm{~s}$ & $1 \mathrm{~Hz}$ \\
\end{tabular}

${ }^{\mathrm{a}} \mathrm{DH}$ DataHawk, $\mathrm{CW}$ cold wire

presented elsewhere (Luce H, Hashiguchi H, Kantha L, Lawrence D, Tsuda T, Mixa T: Concurrent MU radar, UAV and balloon observations of temperature and moisture fine-scale structures during the ShUREX 2015 campaign, in preparation).

\section{ShUREX 2015 campaign}

The ShUREX 2015 campaign was conducted at Shigaraki MU Observatory from June 1 to June 14, 2015, with the goal of obtaining simultaneous measurements of the lower atmosphere in the vicinity of the radar through synergistic use of the UAV and the radar. Particular emphasis was placed on extracting turbulence parameters from the radar and the UAV-borne sensors. Attempts were made to probe interesting structures reachable by the UAV, guided in real and near real time by the radar.

The UAVs were launched from a nearby field roughly $1 \mathrm{~km}$ southwest of and about $50 \mathrm{~m}$ below the radar antenna. Most of the launches were made using a bungee cord, and the battery energy consumed during the climb (typically $2 \mathrm{~ms}^{-1}$ ) limited the maximum altitude attainable to about $4.0 \mathrm{~km}$ (ASL). Balloon launches, in which the UAV is carried aloft by a commonly used weather balloon and cut adrift at a pre-determined altitude, enabling the UAV to reach altitudes in excess of $7.0 \mathrm{~km}$.

High winds aloft and battery power constraints prevented sampling of interesting structures at heights of 5.0 to $7.0 \mathrm{~km}$ in the column (e.g., Fig. 3). However, two balloon launches were made, one (UAV 17) to a height of $5.0 \mathrm{~km}$ on June 11, enabling sampling of the top of a MCT layer at $4.0 \mathrm{~km}$. The second (UAV 19) enabled repeated up and down yo-yo sampling of another but shallower convective layer at a height of $2.0 \mathrm{~km}$ on June 13 .

High winds and rain prevented UAV flights on some days, but a total of 19 UAV flights were made on five of the days (June $5,7,9,10$, and 11) during the campaign (see Table 3). While a large fraction of these involved a spiraling climb and descent over the launch field, several involved different trajectories as shown in Fig. 3. These show the versatility of UAVs in in situ probing of the atmosphere, guided in real or near real time by the radar.

In this overview, we will describe some preliminary results mostly obtained from UAV 5 and UAVs 12 and 17, whose flight paths are shown in Fig. 3. These results demonstrate the good performance of the MU radar in range imaging mode and the potential of UAV measurements. Future work will focus on specific aspects of these and other flights, which can be studied from the datasets. Analyses of some of the data and syntheses of the results are presented in Luce et al. (2017) and (Luce H, Hashiguchi H, Kantha L, Lawrence D, Tsuda T, Mixa T: On the performance of the range imaging technique using UAVs during the ShUREX 2015 campaign, submitted).

UAV 12, on June 9, 2015: detection of UAVs by the MU radar Because the UAVs were flying close to the radar antenna array, they could be detected and monitored, mostly by side lobes of the radar beam pattern. Radar echo contaminations by UAVs are obviously a drawback when comparing atmospheric data but can also be an advantage. They provide the opportunity to calibrate the radar altitude to the altitude provided by the onboard sensors and to synchronize the two datasets. Figure 4c shows an example of high-resolution, time-height image of echo power at vertical incidence on June 9, between 16:30 and 18:00 LT during the UAV 12 flight. Echoes due to the reflection from the UAV appear as a single, thin, and continuous line in the image below the altitude of $3.0 \mathrm{~km}$ ASL from $\sim 17: 00 \mathrm{LT}$ until $\sim 17: 45 \mathrm{LT}$. This is despite the presence of strong atmospheric echoes (the observed atmospheric structures for this flight will not be discussed here). Therefore, it is clearly evident, as expected from theoretical considerations and from earlier applications, that the range imaging processing with the Capon method does not produce any "ghost" echo, i.e., repeated peaks due to some range ambiguities or inappropriate properties. This straightforward result gives extra credence to the existence of atmospheric (echo) layers revealed by the range imaging technique. 

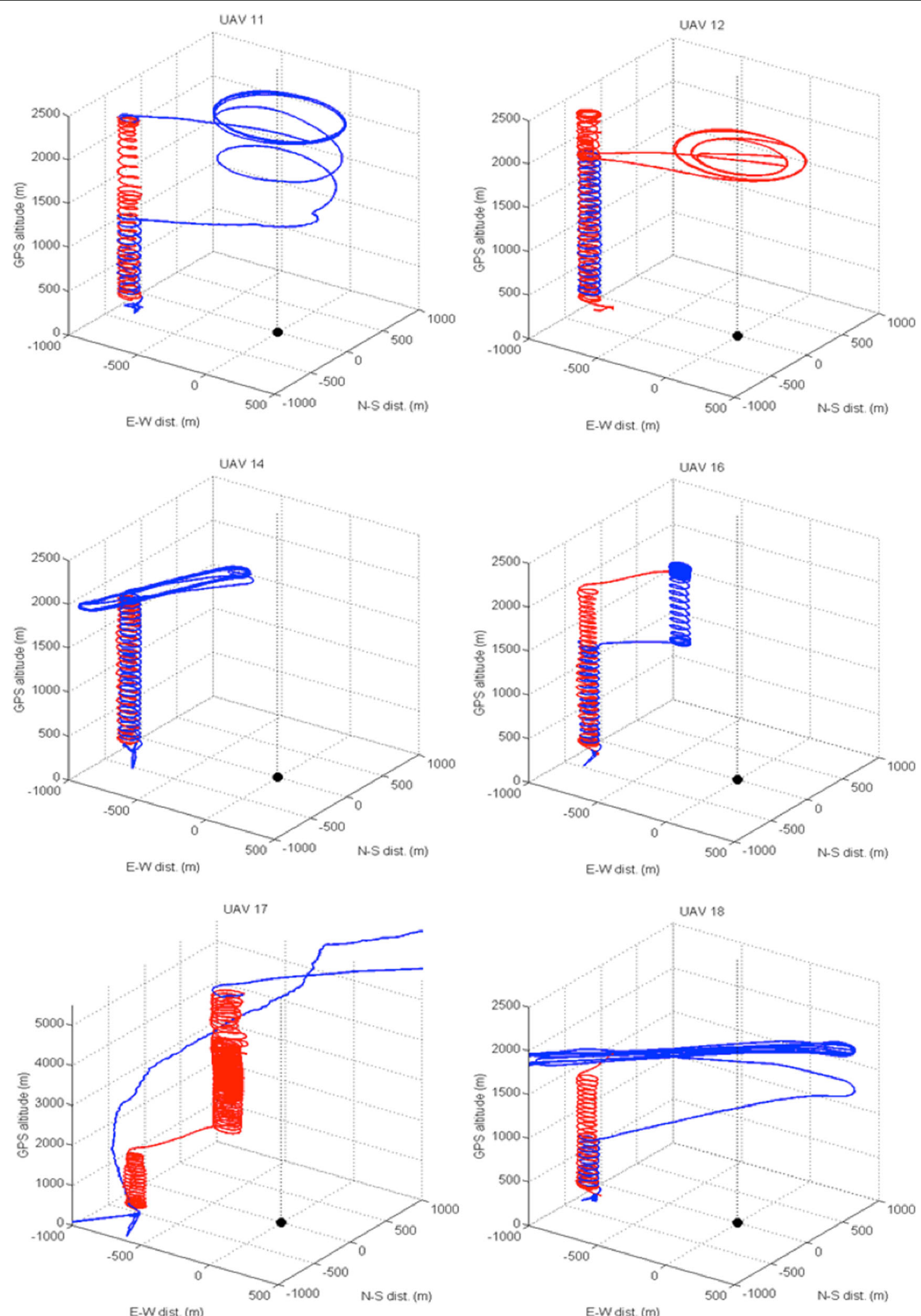

Fig. 3 Trajectories (from top left clockwise) of UAV 11, UAV 12, UAV 16, UAV 18, UAV 17, and UAV 14. Red curves denote ascent 
Table 3 Catalog of science flights during the ShUREX 2015 campaign

\begin{tabular}{|c|c|c|c|c|c|}
\hline Date & UAV\# & Launch time & Max Ht (km) & Launch & Purpose \\
\hline 05_June & 1 & 06_32_13 & 3.0 & Bungee & Over the field \\
\hline 05_June & 2 & 07_48_46 & 3.0 & Bungee & Over the field \\
\hline 05_June & 3 & 09_12_56 & 4.0 & Bungee & Over the field \\
\hline 07_June & 4 & 05_44_00 & 3.7 & Bungee & Over the field \\
\hline 07_June & 5 & 07_14_11 & 4.2 & Bungee & Over the field \\
\hline 07_June & 6 & 09_44_58 & 3.5 & Bungee & Over the field \\
\hline 07_June & 7 & 10_55_36 & 3.9 & Bungee & Over the field \\
\hline 07_June & 8 & 12_55_28 & 3.2 & Bungee & Over the field \\
\hline 07_June & 9 & 15_34_41 & 3.2 & Bungee & Over the field \\
\hline 07_June & 10 & 17_33_43 & 4.3 & Bungee & Over the field \\
\hline 09_June & 11 & 15_46_14 & 2.4 & Bungee & Over the radar \\
\hline 09_June & 12 & 16_55_11 & 2.5 & Bungee & Over the radar \\
\hline 10_June & 13 & 07_28_08 & 0.7 & Bungee & Gravity wave \\
\hline 10_June & 14 & 08_22_20 & 2.0 & Bungee & Gravity wave \\
\hline 10_June & 15 & 10_16_52 & 1.8 & Bungee & Gravity wave \\
\hline 11_June & 16 & 05_32_11 & 2.3 & Bungee & Near the radar \\
\hline 11_June & 17 & 09_23_03 & 5.0 & Balloon & Near the radar \\
\hline 13_June & 18 & 09_34_20 & 1.7 & Bungee & Gravity wave \\
\hline 13_June & 19 & 12_12_43 & 2.7 & Balloon & Long-term survey \\
\hline
\end{tabular}

The range of the UAV echo, after subtracting the radar antenna altitude (i.e., $378 \mathrm{~m} \mathrm{ASL}$ ), should correspond to the distance of the vehicle from the center of the radar antenna array. This distance could be deduced from the GPS measurements made onboard. Because the UAV has small dimensions $(\sim 1 \mathrm{~m})$ compared to the size of the radar volume $(\sim 100 \mathrm{~m})$, it can be considered to be a discrete target unless it is moving with respect to the radar. When circling at almost constant radial distance around the center of the radar antenna, the UAV would therefore appear as a fixed target to the radar. Such a flight configuration (Fig. 3) was used for a while during UAV 12 flight, which was therefore well adapted for evaluating the performance of the range imaging technique in greater detail. Furthermore, a refinement of the altitude calibration of the MU radar could be achieved.

The UAV ascended first near the launching site in a helical pattern of $\sim 120 \mathrm{~m}$ in diameter from the ground up to $\sim 2040 \mathrm{~m}$ ASL at a vertical velocity of $2 \mathrm{~ms}^{-1}$ for $\sim 13 \mathrm{~min}$ (Fig. 4a, b). It was then directed toward the MU radar antenna and flew a series of constant height circles at two slightly different levels centered on the MU radar antenna. During this period, the UAV first made two and a half circles of $\sim 850 \mathrm{~m}$ in diameter at $\sim 2033 \mathrm{~m}$ ASL for about $11 \mathrm{~min}$ from $\sim 17: 11 \mathrm{LT}$ and then made two circles of $\sim 620 \mathrm{~m}$ in diameter at $\sim 2015 \mathrm{~m}$ ASL for about $4.5 \mathrm{~min}$. After these sequences, the UAV passed directly over the radar antenna at around 17:26 LT and exactly over the vertical around 17:29 LT. A bright spot due to the passage of the UAV into the mean beam can be seen at that time in the radar image (Fig. 4c). The UAV went back toward the launching site and made a series of additional circular patterns of $\sim 120 \mathrm{~m}$ in diameter before descending to the ground (see Fig. 3). Between 17:10 LT and 17:30 LT, it was flying at a constant distance from the center of the radar antenna so that the radial velocity of the vehicle (i.e., the velocity component parallel to line of sight of the radar beam) was minimized.

Figure $4 \mathrm{~d}$ shows the GPS distance of the UAV from the radar antenna plotted against time (red line) at a time resolution of $1 \mathrm{~s}$ and the corresponding positions of the Capon peaks manually selected from Fig. 4c. The correspondence between the GPS positions and the radar-derived positions is excellent after a small (and already applied) correction of $+25 \mathrm{~m}$ to the radar altitudes with respect to the nominal altitudes. The Capon processing method thus provides a faithful image of the target with accurate positions, since small distance variations due to the helical trajectory of the UAV (of the order of a few tens of meters or less) could be monitored under the conditions of the experiments. Importantly, the refined altitude calibration of the MU radar was confirmed by most other flights. (Luce $\mathrm{H}$, Hashiguchi $\mathrm{H}$, Kantha L, Lawrence D, Tsuda T, Mixa T: On the performance of the range imaging technique using UAVs during the ShUREX 2015 campaign, submitted) provide a more detailed description of the results with the Capon method and MUSIC algorithm. 


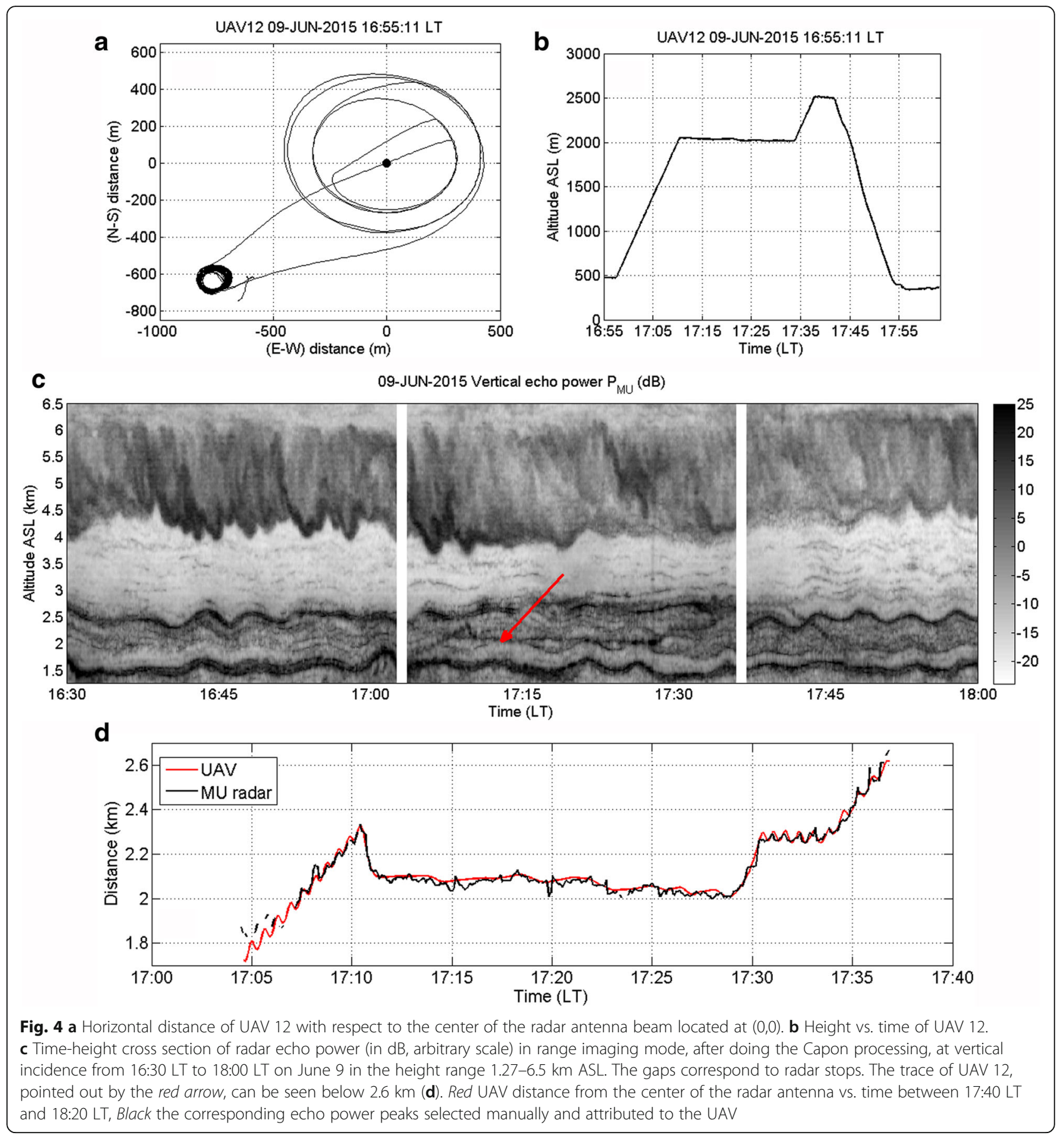

\section{UAV 17 on June 11, 2015: sampling of several turbulent layers}

On June 11, 2015, a UAV was launched by balloon to an altitude of $5.4 \mathrm{~km}$. During that time, it drifted away from the radar and the launch site, so it was flown back to the vicinity of the radar, about $400 \mathrm{~m}$ west of it. The altitude of the UAV vs. time is shown by the black line and is labeled "A1" (ascent 1) in Fig. 5b. Starting from 09:20 LT, the UAV performed an almost continuous spiral descent to an altitude of $2.7 \mathrm{~km}$ to sample a convective layer (MCT) until 09:40 LT. This phase is shown by the blue line and is labeled "D1" (descent 1). Then, it was commanded to ascend to an altitude of $4.0 \mathrm{~km}$ to sample the feature again until 09:49 LT (red line, label "A2," ascent 2). It was finally commanded to execute a spiral descent to $1.9 \mathrm{~km}$ at $\sim 10: 05 \mathrm{LT}$ before heading over to the launch field and executing a spiral descent back to the ground (358 m ASL) (green line, label “D2," descent 2). 


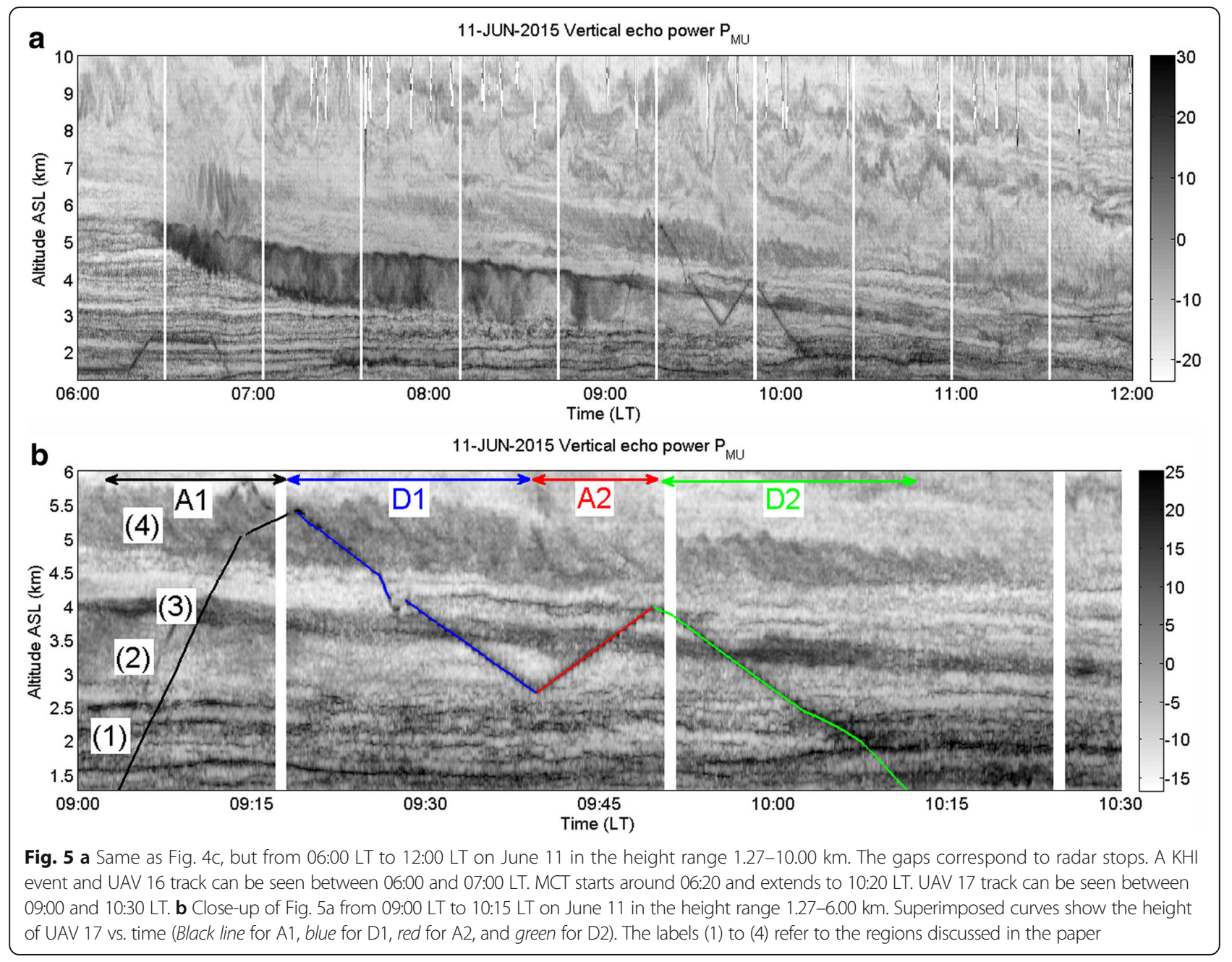

\section{MU radar measurements}

Figure 5a shows a time-height plot of the radar echo power during UAV 17 at vertical incidence and in range imaging mode from 06:00 LT to 12:00 LT on June 11 in the height range 1.27 to $8.00 \mathrm{~km}$. On that day, one can see KHI billows occurring at a mean altitude of $6.5 \mathrm{~km}$ and extending roughly $1 \mathrm{~km}$ top to bottom. The billows lasted from 06:31 LT to 06:55 LT. Immediately below, mid-level cloud-base convection (see Kudo 2013; Wilson et al. 2014; Kudo et al. 2015 for a description of MCT) was initiated at a height of $\sim 5.5 \mathrm{~km}$ at around 06:16 LT. Incidentally, the signature of another UAV (UAV 16) launched prior to UAV 17 can be seen from 06:20 LT to $06: 50$ LT but UAV 16 did not fly high enough to reach the deep turbulent layers. The MCT layer expanded rapidly to span 5 to $5.5 \mathrm{~km}$ by 07:30 LT and descended to lower altitudes and thickened. Turbulence inside the layer weakened by the time UAV 17 could be launched to sample it. Nevertheless, useful data were collected in the decaying MCT layer, and the ability of the sensors on the UAV to sample atmospheric structures like these was demonstrated. Synergistic use of UAVs and MST radars such as the MU radar was also demonstrated, since the UAV could be commanded in real or near real time to sample the structure, guided by radar images.

Figure 5b shows a close-up of Fig. 5a centered around UAV 17 flight. As can be seen from the figure, the MCT layer, region labeled (2), was sampled twice. The label (1) refers to an altitude range showing persistent fine structures; the label (3), a strong echo layer at the interface between the convective layer and cloud; and label (4), an echo layer with more or less well-defined braided structures believed to be the result of a KHI in the cloud. Despite strong atmospheric echoes, UAV echoes can be distinguished around $2.0 \mathrm{~km}$ ASL between $~ 17: 00$ and 17:50 LT below a deep and persistent turbulent layer (identified as MCT and centered around $5.0 \mathrm{~km}$ ).

\section{UAV measurements}

Only the PTU data taken by the iMET sensors, the TU data taken by slow response sensors on the UAV (denoted by $\mathrm{DH}$, see Table 2), and the temperature data 
taken by the fast response cold wire (denoted by CW) are valid during the initial ascent by balloon, since the UAV was carried aloft by the balloon and not flying under its own power. Therefore, the velocity data are invalid during this phase (A1). Figure 6 shows time series of GPS altitude, temperature, and relative humidity obtained from the three sets of onboard sensors during UAV 17. The blue curves correspond to the sensors on iMET, the red curves to the DH sensors, and the black curve for temperature to the $\mathrm{CW}$ sensor. There is general agreement between the various time series, except for the fact that there is an offset of about $0.8{ }^{\circ} \mathrm{C}$ in the $\mathrm{DH}$ and $\mathrm{CW}$ temperature data, compared to iMET temperature data and $-15 \%$ in the $\mathrm{DH}$ humidity data, compared to the iMET humidity data. However, apart from these biases, the overall trend appears to be reproduced well by all sets of sensors.

Figure 7 shows temperature and relative humidity profiles during UAV 17. The blue curve corresponds to the descent D1, the red curve to ascent A2, and the black curve to descent D2. The initial ascent of the UAV borne by the balloon has been omitted for clarity. Also, in the top two panels, the profiles have also been offset to the right by $5{ }^{\circ} \mathrm{C}$ for temperature and $50 \%$ for humidity. In the top left panel, the leftmost profile refers to the iMET data, with the DH profile offset to its right. This is followed by the profile to its right. In the top right panel, only the iMET and DH humidity profiles are shown (there is no corresponding third high-resolution humidity profile). Once again, the agreement between the various sets of sensors is quite good, in general, except for the offsets mentioned above.
When properly calibrated, the three sensor sets capture trends in the data quite well, with slight variations between different datasets highlighting the importance of synergistic use of the instruments to determine the most accurate measurement when disagreements arise. The CW temperature has a much smaller time constant than the iMET temperature (see Table 2) so the radiosonde temperature minima from 2400 to $2700 \mathrm{~s}$ and 3000 to $3300 \mathrm{~s}$ (see Fig. 6) are likely artifacts, since they do not occur in the higher-fidelity $\mathrm{CW}$ data. The iMET humidity sensor has a smaller time constant than the DH sensor, so the iMET humidity minima from 3700 to $4000 \mathrm{~s}$ are likely real, since the DH humidity sensor cannot respond as quickly to changes in the ambient air as iMET humidity sensor can. In cases where the iMET temperature and humidity sensors experience simultaneous excursions that do not occur in the DH data, it is likely to be an error in both radiosonde sensors, since they are coupled on the same sensor array and occupy the same circuitry. The GPS altitude has higher "local" uncertainty than the pressure altitude, making pressure altitude the more accurate measurement when the two disagree over the short term. However, pressure altitude has longterm errors over large altitude ranges that GPS does not exhibit. Relative benefits of different sensor datasets have therefore been used to produce a more accurate composite set of measurements.

The iMET temperature exhibits spikes (e.g., at 2.5 and $3.2 \mathrm{~km}$, Fig. 7), perhaps due to the impact of water particles because of its exposed location on the UAV. These spikes are not present in the other two temperature sensors and are removed before analysis. The bottom
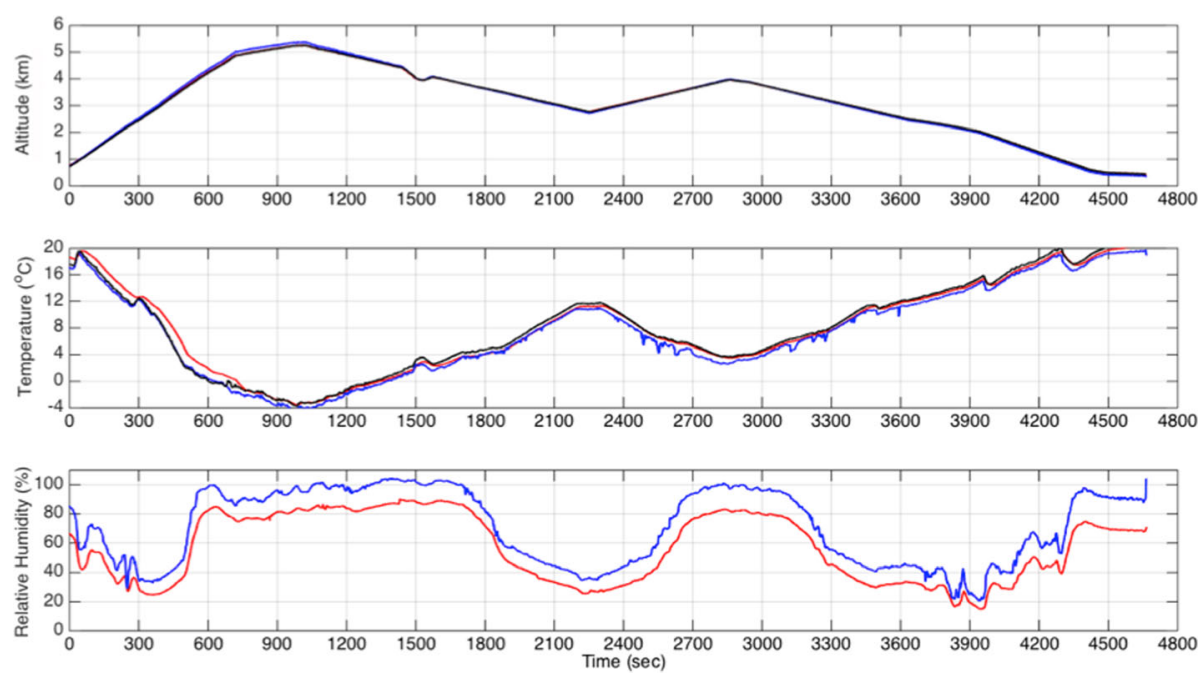

Fig. 6 The altitude (top panel), temperature (middle pane), and relative humidity (bottom panel) as a function of time for UAV 17. In the temperature and humidity plots, the blue curve corresponds to the sensors on iMET, the red curve to the DH sensors, and the black curve to the cold wire (CW) sensor. There is general agreement between the various sensors, except for the fact that an offset of $-0.8^{\circ} \mathrm{C}$ had to be applied to the DH and CW temperature data and $+15 \%$ to the $\mathrm{DH}$ humidity data. The altitude plot shows altitude derived from the $\mathrm{DH}$ (black) and iMET (blue) pressure sensors 

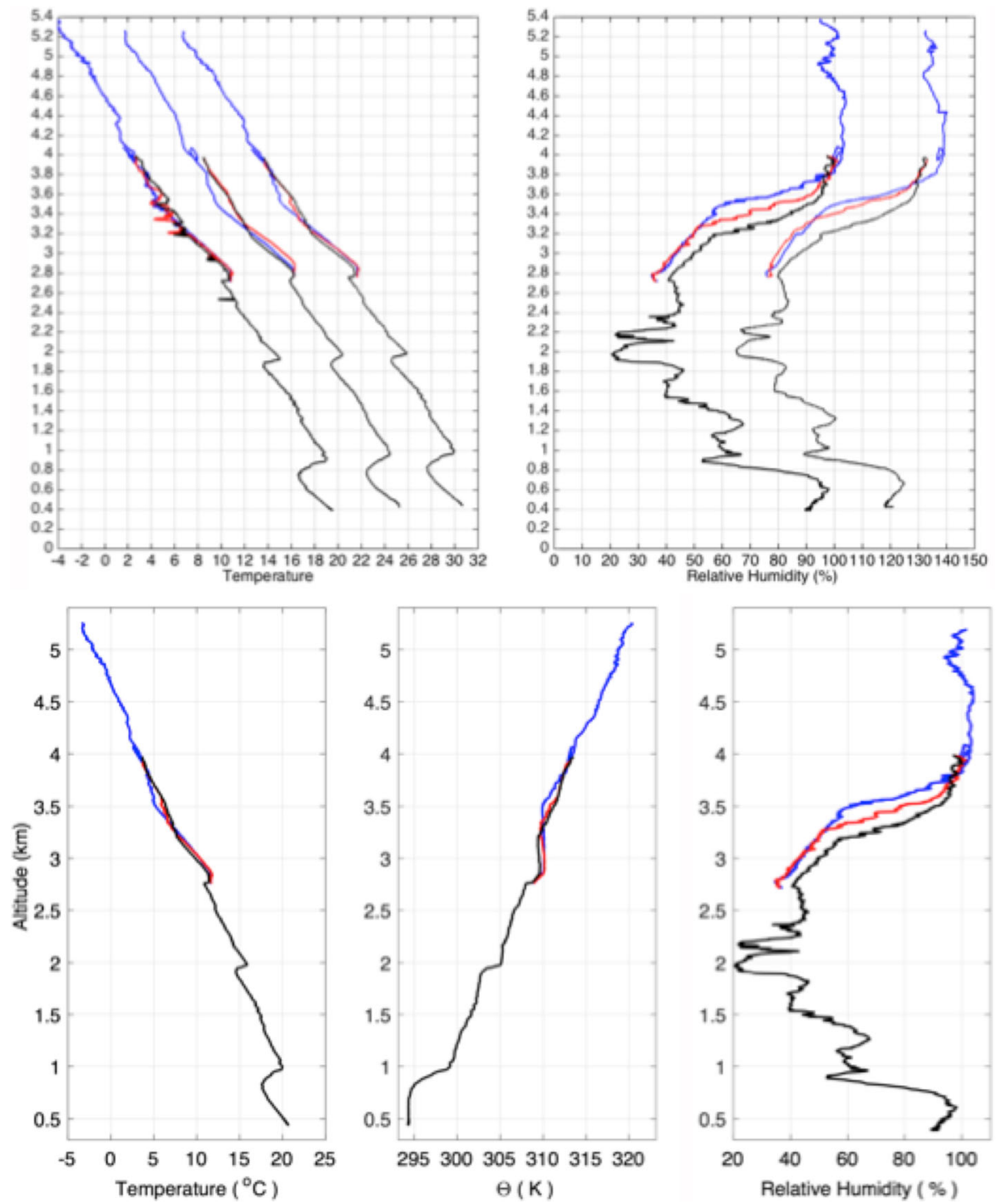

Fig. 7 The top left panel shows the temperature profiles and the top right panel, relative humidity profiles during the UAV 17 flight. The blue curve corresponds to descent D1, the red curve to ascent A2, and the black curve to descent D2. The initial ascent (A1) borne by the balloon has been omitted for clarity. Also for clarity, the profiles have been offset to the right by $5{ }^{\circ} \mathrm{C}$ for temperature and $50 \%$ for humidity. In the top left panel, the first profile refers to the iMET sensor data, with the DH profile offset to its right, which is followed by the CW profile to its right. In the top right panel, the relative humidity profile is from the iMET sensor followed to its right by that from the DH sensor. The bottom three panels show composite profiles of temperature (bottom left) and relative humidity (bottom right) from the three sets of sensors and the potential temperature derived from them (bottom middle). Once again, the blue curve corresponds to descent D1, the red curve to ascent A2, and the black curve to descent D2

three panels of Fig. 7 show the composite profiles of temperature, relative humidity and potential temperature after quality control, which selects the best data for each variable at each height.

\section{Retrieval of atmospheric parameters From radar measurements}

Comparison of estimates of radar- and UAV-derived atmospheric parameters can be made in various ways due to the different nature of the measurements. A UAV does not provide truly vertical profiles as instrumented towers (or balloons) do, but time series along inclined or even horizontal paths. Therefore, the most intuitive way would be to reconstruct time series of radar-derived atmospheric parameters along the UAV path in a manner identical to Fig. 6. Another approach, used here, is to reconstruct pseudo-vertical profiles along the inclined path of the UAV (only possible when the UAV is not flying horizontally) so that a standard description of the results in terms of altitude range can be made. Once time-height 


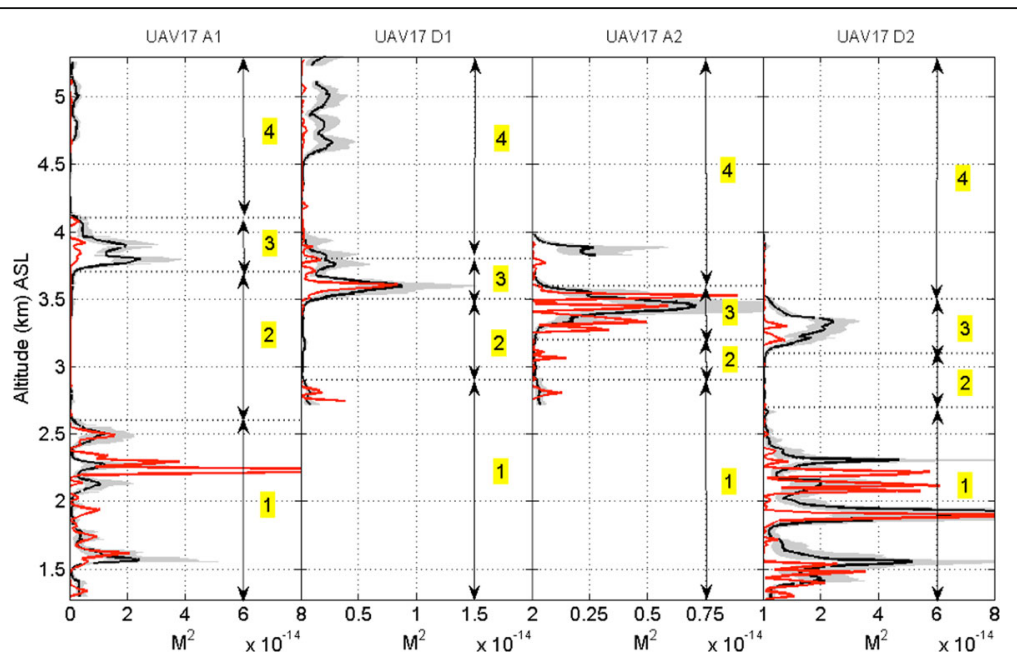

Fig. 8 Comparisons between $M_{\text {UAV }}^{2}$ (red) and $M_{\text {radar }}^{2}$ (black) profiles in linear scales for A1, D1, A2, and D2 flight phases of UAV17. The labels (1) to (4) refer to the region emphasized in Fig. $5 \mathrm{~b}$. Horizontal scales have been adapted to the dynamics of the values

cross sections of a given parameter are made from radar data (such as echo power in Fig. 5), a radar data sample for each height and time of the UAV, when the UAV is moving up or down, is obtained from a linear interpolation of the radar-derived values at the height of the UAV. Selecting an "instantaneous" radar estimate is possible, but it is affected by statistical errors and possible remaining outliers that could bias the comparisons. For the present work, it was arbitrarily decided to time average over $2 \mathrm{~min}$ (i.e., $\pm 1 \mathrm{~min}$ the UAV time), corresponding to about the average of a maximum of 30 consecutive time samples.

\section{Vertical gradient of the generalized potential refractive index}

An example of comparison results using the methodology described above is shown in Fig. 8 for the four ascending and descending segments of UAV 17 flight. The atmospheric parameter shown in this figure is the
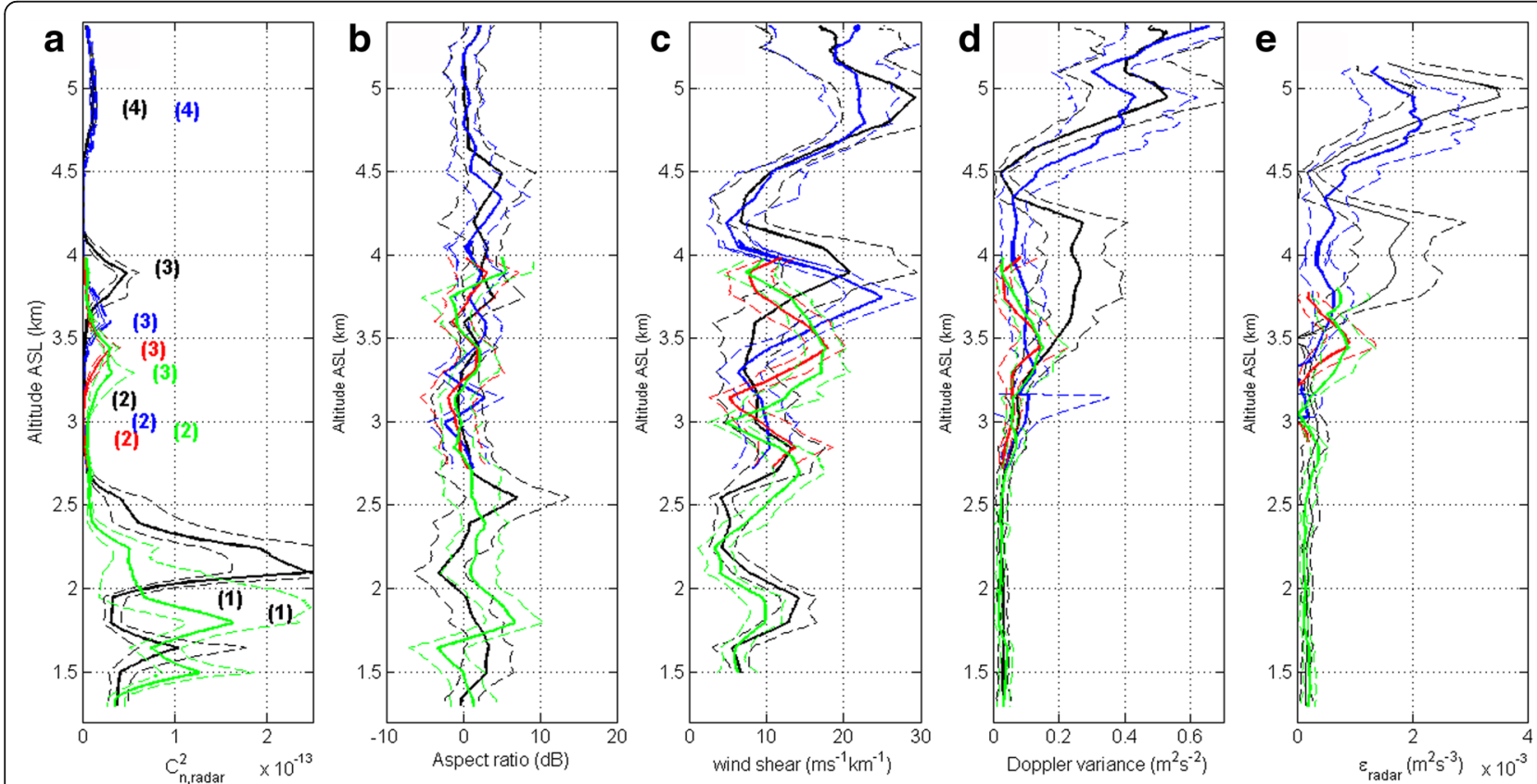

Fig. 9 a-e Vertical profiles of parameters estimated from the MU radar measurements during UAV 17. The leftmost panel shows $C_{n, \text { radar }}^{2}$ and the rightmost panel shows $\varepsilon_{\text {radar }}$ using Eq. (3). The labels (1) to (4) shown in the leftmost panel refer to altitude ranges indicated in Figs. 5b and 8 . The black curve corresponds to A1, blue to D1, red to A2, and green to D2 (see Fig. 5b) 


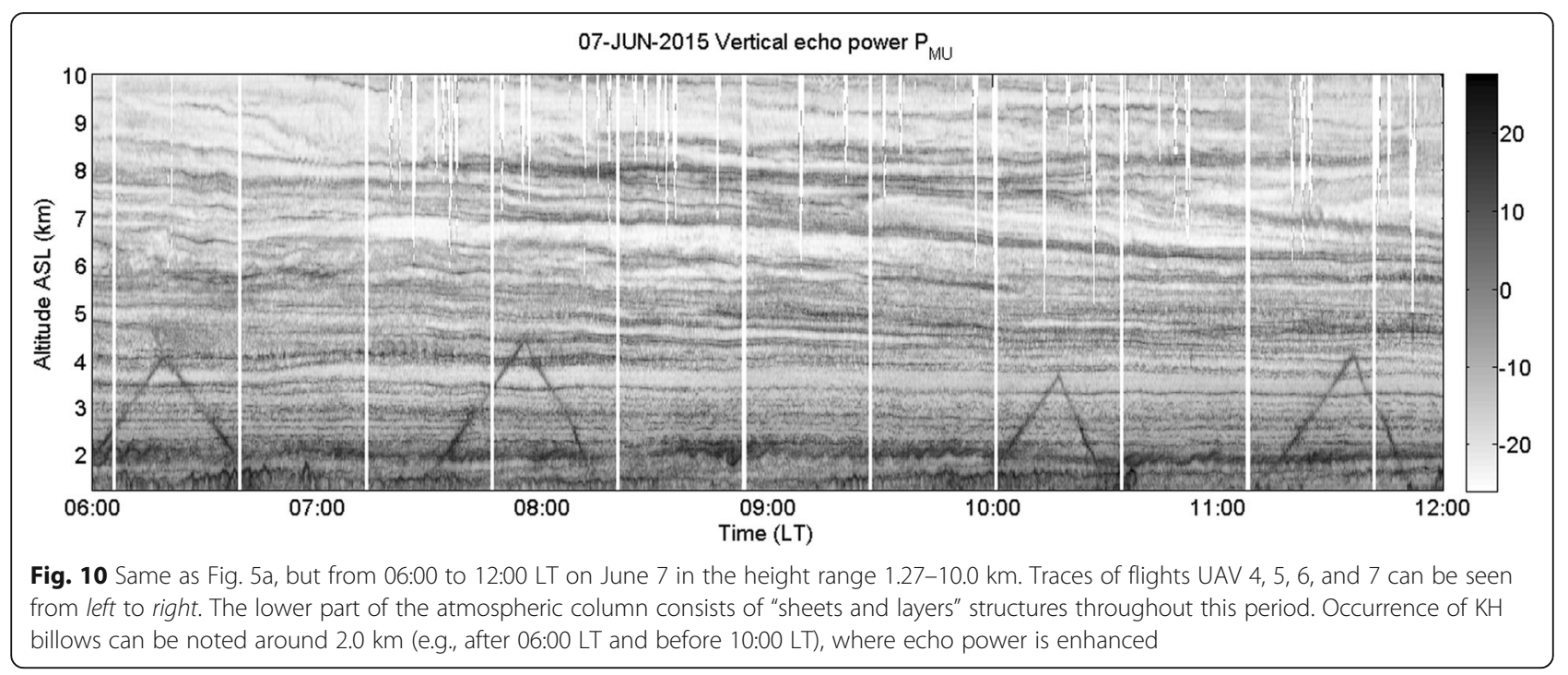

vertical gradient of the generalized potential refractive index $M^{2}$ (Ottersten 1969). For unsaturated conditions, it is defined as

$$
M=77.16 \times 10^{-6} \frac{P}{T}\left(\frac{N^{2}}{g}+\frac{15500 q}{T}\left[\frac{N^{2}}{g}-\frac{1}{2 q} \frac{d q}{d z}\right]\right)
$$

where $N^{2}=\frac{g}{T}\left(\frac{d T}{d z}+\Gamma\right)$ is the square of the buoyancy frequency, $\Gamma$ is the dry adiabatic lapse rate $(\mathrm{K} / \mathrm{m}), T$ is the temperature $(\mathrm{K}), p$ is the pressure $(\mathrm{hPa}), q$ is the specific humidity $(\mathrm{g} / \mathrm{g})$ and $g$ is the gravitational acceleration.

It is well known that the radar echo power $P$ corrected for range attenuation effects (i.e., $P \times z^{2}$ ) is closely related to $M^{2}$ at vertical incidence, at least at resolutions of $\sim 150 \mathrm{~m}$ and $60 \mathrm{~min}$, coarser than in the present study (see Hooper et al. 2004; Luce et al. 2007 and references therein). Here, $M_{\mathrm{UAV}}^{2}$ was estimated from Eq. (1) at a vertical resolution of $20 \mathrm{~m}$, using PTU data collected from the iMET sensors and $M_{\text {radar }}^{2}=K P z^{2}$, where $K$ is a coefficient
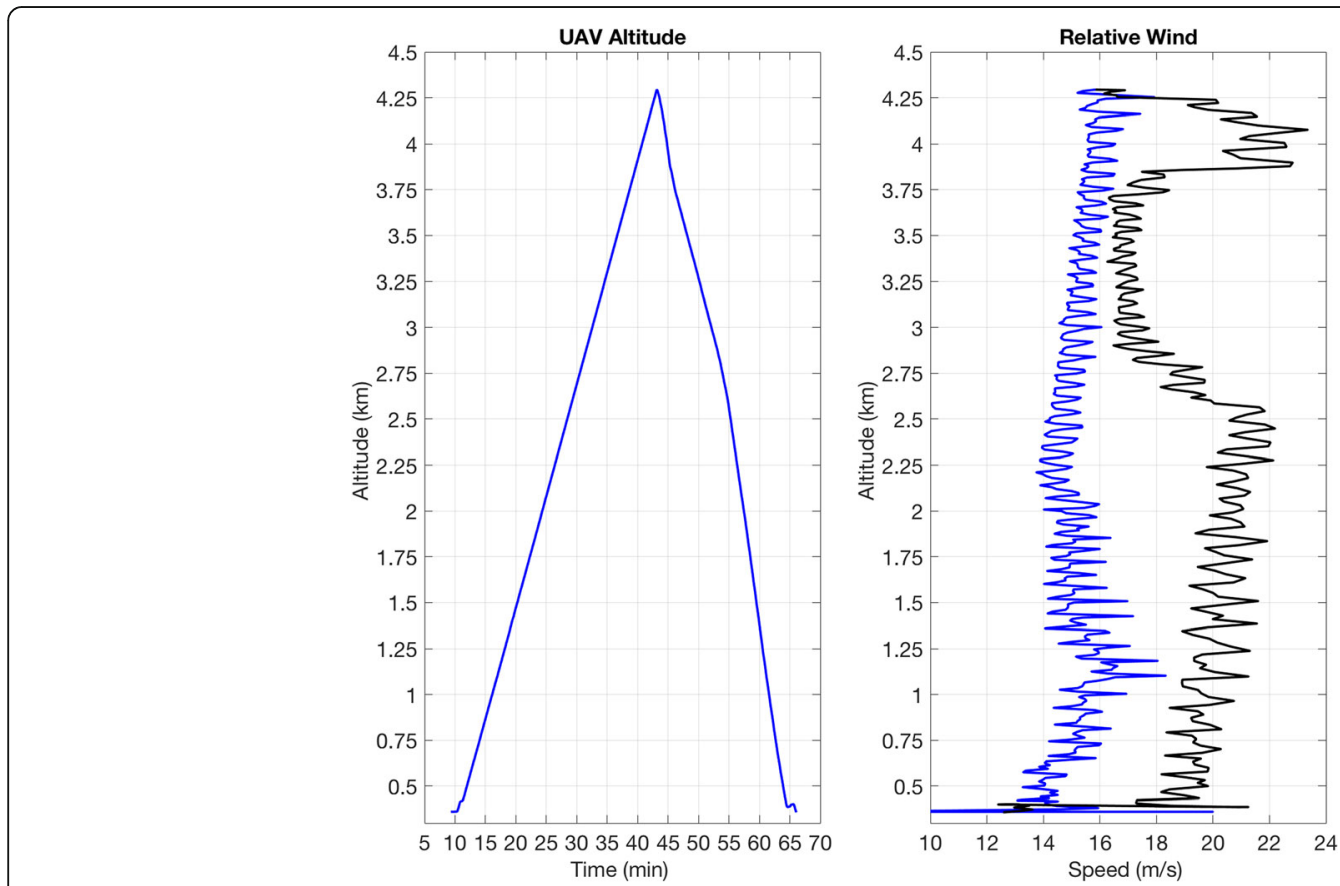

Fig. 11 (Left panel) Altitude of the UAV for flight UAV 5 as a function of time. (Right panel) Speed of the UAV relative to the ambient air. Ascent is shown in blue and descent in black 
taken to be consistent with the value used by Luce et al. (2007) from radar-balloon comparisons of $M^{2}$ at stratospheric heights. A detailed description of the methods used and discussions about the relationship between $M^{2}$ and radar echo power are given by Luce et al. (2017).

Figure 8 shows a good overall agreement between the characteristics of the peaks of $M_{\mathrm{UAV}}^{2}$ and $M_{\mathrm{radar}}^{2}$. In particular, the strong maxima of $M_{\text {radar }}^{2}$ usually coincide with maxima of $M_{\mathrm{UAV}}^{2}$ with the same order of magnitude in regions (1), (2), and (3). The ratio is often close to 1 and generally less than 2 or 3 , except during the first ascent (A1) around $2.3 \mathrm{~km}$ where $M_{\mathrm{UAV}}^{2}$ is much stronger than $M_{\text {radar }}^{2}$. The difference in region (4), where a KH layer is observed inside the cloud, might be significant due to the fact that $M_{\mathrm{UAV}}^{2}$ given by Eq. (1) is not valid for saturated conditions. Luce et al. (2017) present a more thorough analysis and interpretations of similar results obtained during a series of six UAV flights on June 7, 2015.

When possible, the contaminations produced by UAV echoes were manually removed, after editing each Doppler spectrum for each beam direction. Each portion of the contaminated spectra was replaced by an averaged value of noise power density before recalculating the primary parameters (power, Doppler shift, spectra width). This manual procedure also permitted removal of additional artifacts (such as ground clutter, bird or airplane echoes, and other contaminations from unknown origins) as far as possible.

\section{Radar aspect ratio}

This is defined as the difference $P_{v}(\mathrm{~dB})-P_{o}(\mathrm{~dB})$ at the range resolution of $150 \mathrm{~m}$, where $P_{v}$ is the radar echo power at vertical incidence and $P_{O}$ is the average in $\mathrm{dB}$ of the radar echo powers measured from the five oblique directions (see Table 1), $10^{\circ}$ off zenith, and linearly interpolated at the altitude of the vertical sampling. The possible azimuthal dependence of the radar echo power has not been considered here. The aspect ratio parameter is useful for identifying isotropic Bragg scatter from turbulence. Indeed, an aspect ratio close to $0 \mathrm{~dB}$ is a very strong indication that isotropic turbulence at the Bragg scale fills the radar resolution volumes. On the contrary, large and positive values should result from anisotropic backscattering mechanisms.

\section{Horizontal wind and vertical shear}

Horizontal winds were estimated from radial winds measured from the six beam directions. The winds were calculated at the range resolution of $150 \mathrm{~m}$ and at the height sampling of the vertical beam. The vertical shear

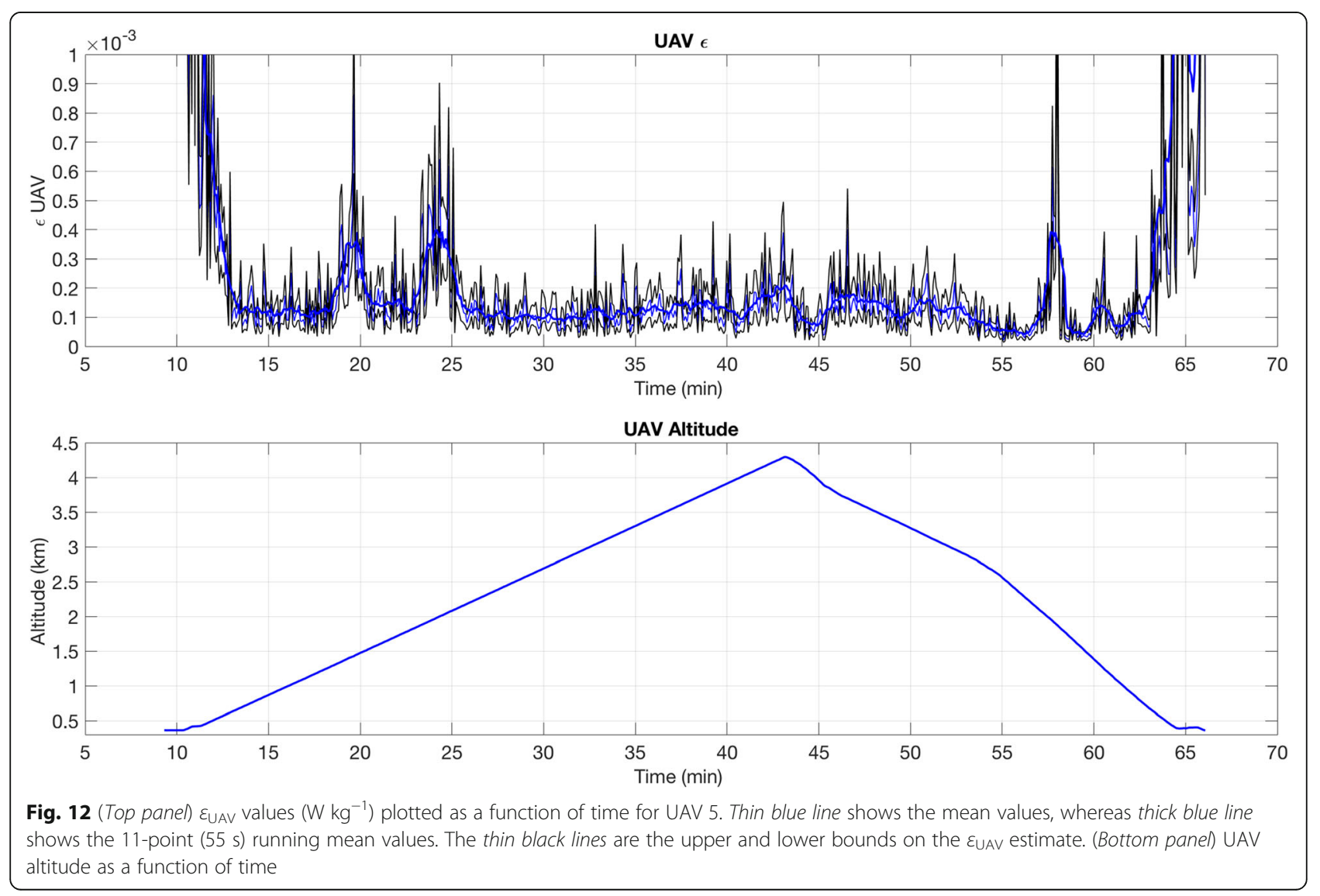


of horizontal wind was then estimated using a two-point symmetric quotient scheme.

The methods for estimating wind and wind shear are based on the hypothesis that the wind field is homogeneous at least over horizontal distances covered by the vertical and oblique beams (i.e., about $1-2 \mathrm{~km}$ at the altitude range of 3-5 km). This hypothesis fails when wind field perturbations occur at horizontal scales of $\sim 1 \mathrm{~km}$ or less, due to $\mathrm{KH}$ instabilities for example. Therefore, the background wind and wind shear can only be obtained after time averaging (30 min or $1 \mathrm{~h}$ is commonly used).

\section{Variance of Doppler peak}

As discussed by Wilson et al. (2014) for example, the variance of Doppler peaks corrected for non-turbulent broadening effects, hereafter denoted by $\sigma_{\text {turb }}^{2}$, can be considered to be an estimate of the variance $\left\langle w^{\prime 2}\right\rangle$ of the vertical wind perturbations produced by isotropic turbulence, at least as a first approximation in regions associated with isotropic backscatter. Various biases in $\left\langle w^{\prime 2}\right\rangle$ mainly due to radar weighting functions and time averaging effects were mentioned by Wilson et al. (2014) and more thoroughly described in the references therein. The variance $\sigma_{\text {turb }}^{2}$ estimated from data collected at vertical incidence can be an underestimate due to the contribution of possible specular reflectors. This eventuality can be easily tested from the radar aspect ratio, which is large in that case. However, when echoes are isotropic, data collected at vertical incidence provide the best estimate of $\sigma_{\text {turb }}^{2}$ because the beam broadening effect is the sole dominant source of biases and can be easily removed. The beam broadening of the Doppler peaks is proportional to the background wind speed, estimated in practice after a time averaging over $\sim 30 \mathrm{~min}$. The method used for removing the beam broadening effect can be found in Wilson et al. (2014, Section 3.2). Other estimates of $\sigma_{\text {turb }}^{2}$ can be obtained from Doppler spectra measured with the $10^{\circ}$ off-vertical beams, but wind shear is an additional factor in beam broadening (e.g., Gossard and Strauch 1983; Doviak and Zrnic 1984; Fukao et al. 1994).

\section{Kinetic energy dissipation rate}

A common expression for the turbulent kinetic energy (TKE) dissipation rate is given by (e.g., Hocking 1983; Fukao et al. 1994):

$$
\varepsilon_{\text {radar }}=0.46 N \sigma_{\text {turb }}^{2}
$$

where $N$ is the Brünt-Vaïsälä frequency. This expression is valid only if outer scales of turbulence are or
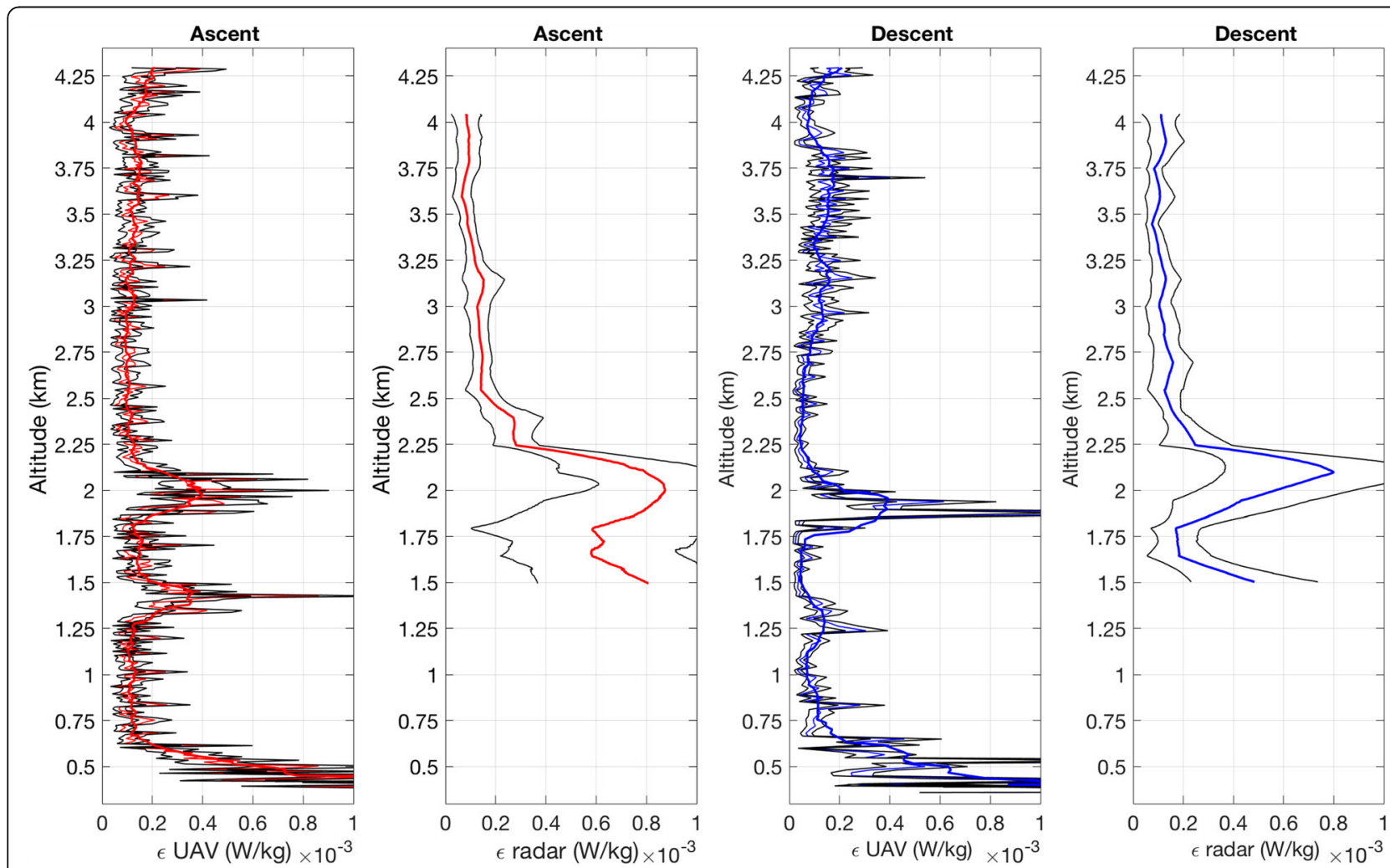

Fig. 13 Vertical profiles of $\varepsilon_{U A V}$ and $\varepsilon_{\text {radar }}$ as a function of altitude for UAV 5 during ascent (first two panels) and descent (last two panels). Thick lines show 11-point (55 s) running means. The thin black lines show the lower and upper bounds on the estimated $\varepsilon$ values (see text for more details) 
the same order of magnitude as the dimensions of the radar volume (see, e.g., White et al. 1999). The purpose of the present work is not to evaluate the validity of Eq. (2) but to provide a preliminary estimate of TKE dissipation rate from radar data using the standard approach. The relevance of Eq. (2) and other expressions given in the literature will be studied in subsequent works.

\section{Turbulence refractive index structure parameter $C_{n \text {,radar }}^{2}$}

The refractive index structure parameter $C_{n}^{2}$ (or a pseudo $C_{n}^{2}$ if the radar returns are not the result of isotropic Bragg scatter), hereafter denoted as $C_{n \text {,radar }}^{2}$, is estimated from radar echo power measured at oblique incidence $\left(10^{\circ}\right.$ off zenith, toward north or east). The power is converted into $C_{n \text {,radar }}^{2}$ using a theoretical (but uncalibrated) radar equation. At present, these profiles should therefore be considered as indicative only. The studies made from ShUREX data also aim to improve $C_{n, \text { radar }}^{2}$ estimates from radar data.

Figure 9 shows reconstructed profiles from radar measurements of various parameters for the segments A1, D1, A2, and D2 of flight UAV 17. Figure 9a shows $C_{n \text {,radar }}^{2}$ profiles with enhanced values at the bottom and top of the MCT layer (region 2). The enhanced values in region 3 (near the cloud base) descend with time. Another flat peak is observed at the height of the KHI layer, region 4 . The corresponding aspect ratio profiles shown in Fig. 9b exhibit values close to $0 \mathrm{~dB}$ especially within the MCT layer (region 2), suggestive of isotropic turbulence. The wind shear profiles (Fig. 9c) show maxima near the cloud base descending with time similar to the peaks of $C_{n, \text { radar }}^{2}$. Another strong shear region is observed at the height of the $\mathrm{KH}$ layer (region 4) and is associated with enhancement of Doppler variance (Fig. 9d). Figure $9 \mathrm{e}$ also shows enhanced $\varepsilon_{\text {radar }}$ in the same range. All these features are very consistent with KHI (shear)induced turbulence in region 4 . The Doppler variance and $\varepsilon_{\text {radar }}$ are also enhanced at the top portion of MCT and at the interface layer.

\section{From UAV measurements Air refractive index}

An in situ estimate of the air refractive index $n$ is computed using the DataHawk temperature $T$ (in ${ }^{\circ} \mathrm{C}$ ), pressure $P$ (in mbar), and relative humidity $u$ (in \%) and applying the standard atmosphere equations of state (Holton 2004):

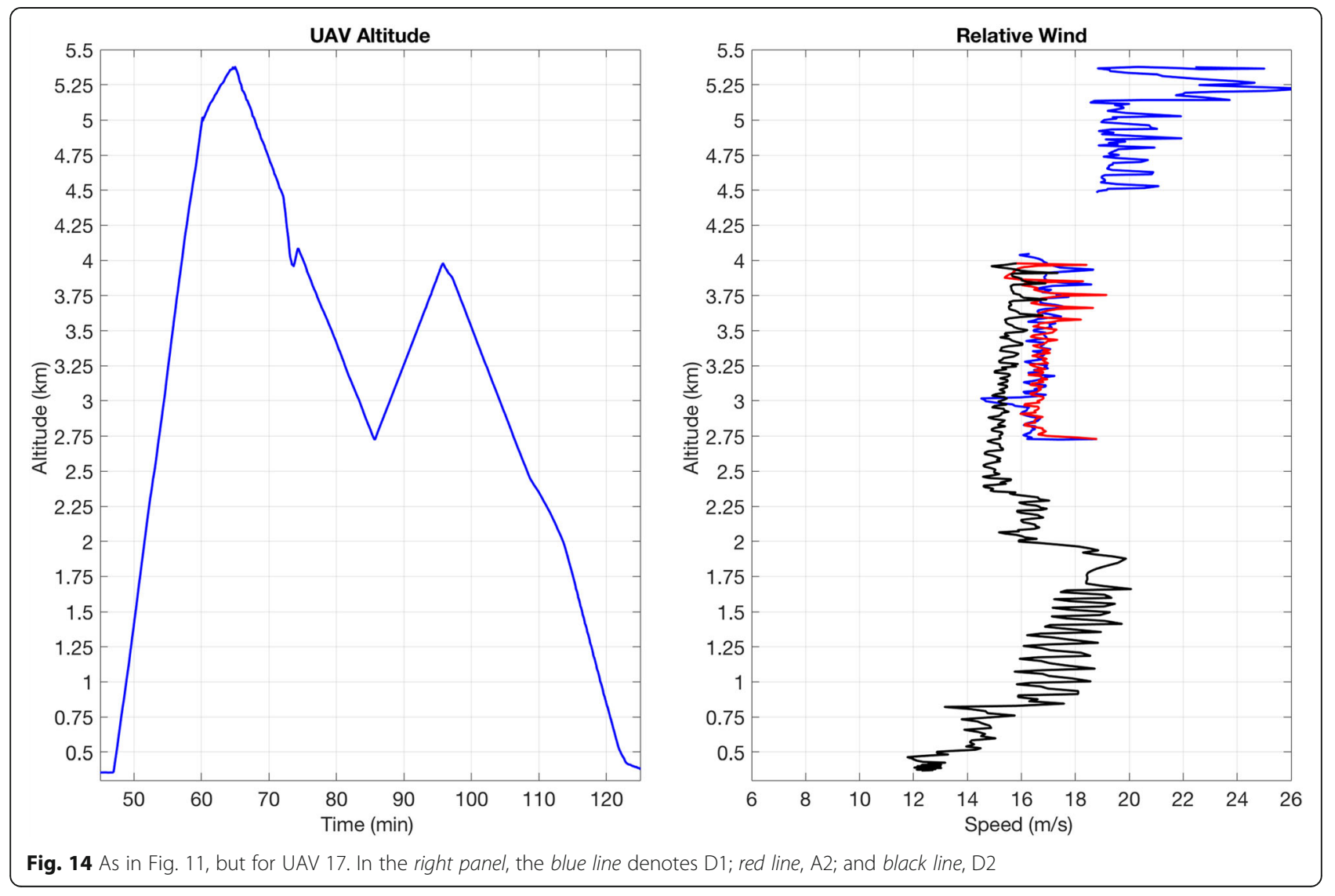




$$
\begin{aligned}
& N=\frac{77.6}{T+273.11}\left(P+\frac{48.11 u e_{s}}{T+273.11}\right) \\
& e_{s}=6.112 \exp \left(\frac{17.6 T}{T+243.5}\right) \\
& n=1+N \times 10^{-6}
\end{aligned}
$$

where $e_{\mathrm{s}}$ is the saturation water vapor pressure. The barometric pressure $P$ is provided by a commercial sensor (MS5611-01BA03) with a resolution of $0.012 \mathrm{hPa}$ and an accuracy of $\pm 1.5 \mathrm{hPa}$, with a time constant of $8.5 \mathrm{~ms}$. It is sampled at $100 \mathrm{~Hz}$. By interpolating the slower humidity measurement, an estimate of $n$ is made at $100 \mathrm{~Hz}$.

\section{Pressure altitude}

In addition to the altitude given by the GPS sensor on the UAV, pressure altitude (in meters ASL) can also be computed from barometric pressure sensor on the UAV, using the lowest tier in the standard atmosphere model

$$
h=44330\left(1+\left(\frac{P}{P_{0}}\right)^{0.190284}\right)
$$

where $P_{0}=1013.25 \mathrm{hPa}$ at sea level. The pressure altitude is used for flight control because GPS altitude can exhibit anomalies of as much as tens of meters, making the UAV flight erratic. However, while the pressure altitude is smoother, it can have large-scale inaccuracy of as much as 300 to $400 \mathrm{~m}$ over $4 \mathrm{~km}$ altitude. Post-flight corrected altitudes are therefore used. These are obtained by fitting a smooth curve (3rd order polynomial) to a pressure altitude vs. GPS altitude plot. Then the pressure altitudes are corrected using this polynomial, producing a smooth altitude that matches GPS over the entire altitude of the flight to within about $20 \mathrm{~m}$ everywhere (ignoring GPS bumps). The corrected pressure altitude does not have local GPS anomalies.

\section{Dissipation rates and structure parameters}

Dissipation rates of turbulent kinetic energy (TKE) $\varepsilon$ and turbulent temperature variance $\chi$, as well as structure parameters $C_{T}^{2}$ and $C_{n}^{2}$, are estimated via spectral

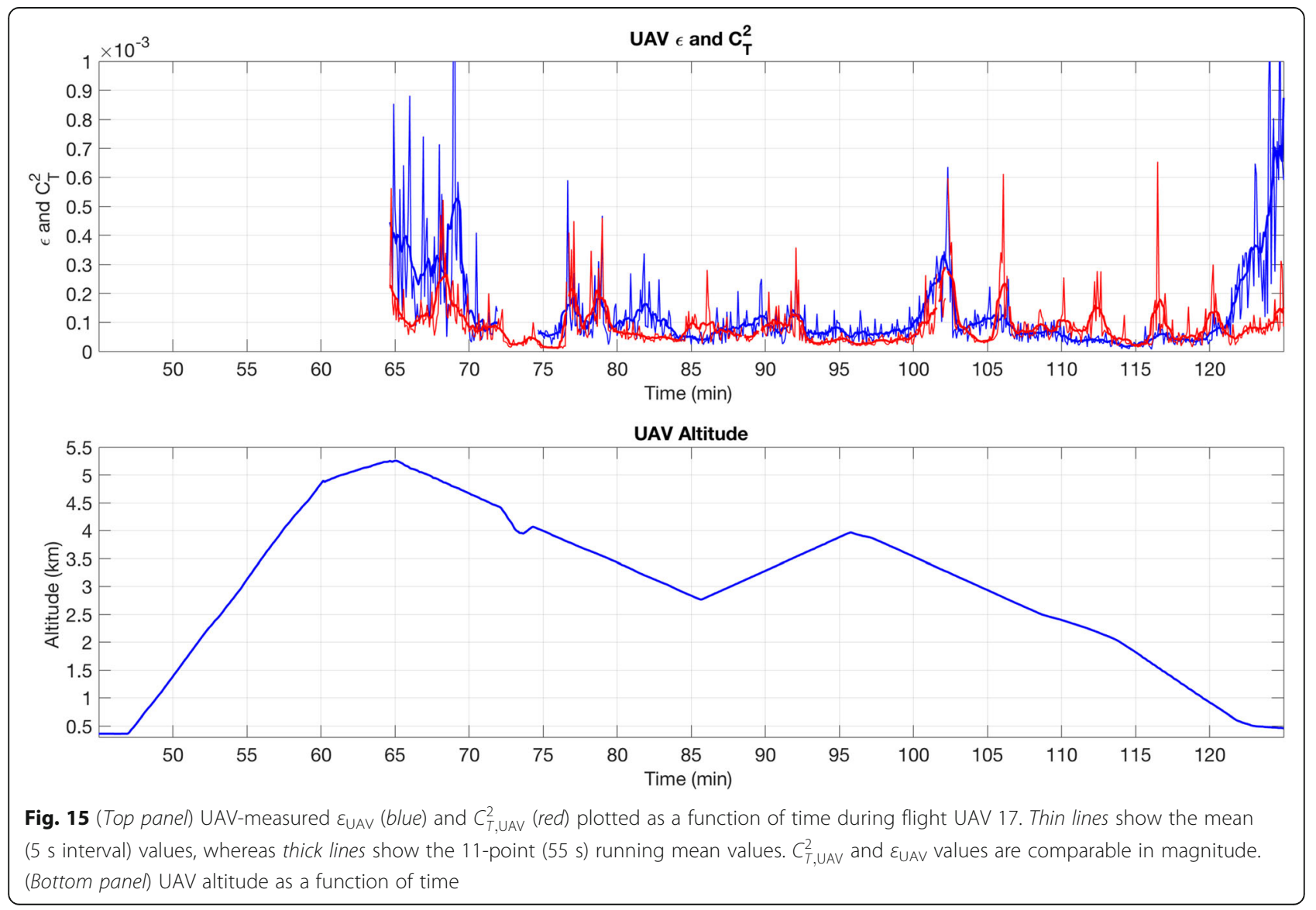


analysis of time series measured above, following Frehlich et al. (2003). Successive 5-second time records are extracted, linearly detrended, and weighted with a 500-point Hanning window. The power spectral density of each record is computed according to

$$
P_{x}\left(f_{k}\right)=2 T\left|\sum_{n=0}^{N-1} x\left(t_{n}\right) e^{-i f_{k} n}\right|^{2}
$$

where $x$ is the time series of interest, $f_{k}=2 \pi k / N, k \in[0$, $N-1$ ], are frequency points with $N$ points in the time record, and $T=N \Delta t$ is the record length.

In turbulent conditions, the power spectral densities are expected to be of the form $P_{x}(f)=c_{x} f^{-5 / 3}$ in the inertial subrange. The parameter $c_{x}$ for that data record is estimated by computing the mean of $P_{x}(f) f^{5 / 3}$ over a suitable range of frequencies so as to avoid aircraft motion artifacts at the lower frequencies and the signal noise floor at higher frequencies. In addition, periodic artifacts from high-frequency structural vibrations were removed from the mean calculation by retaining only the spectral components that did not protrude above 1.5 times the initial mean level.

For estimation of $\varepsilon$, hereafter denoted as $\varepsilon_{U A V}$, the pitot airspeed time series (relative to the wind and hence making use of the Taylor frozen turbulence hypothesis) was used for $x$, resulting in the estimate

$$
\varepsilon_{\mathrm{UAV}}=\left(c_{a s} / 0.146169\right)^{3 / 2} / U
$$

(in units of $\mathrm{m}^{2} \mathrm{~s}^{-3}$ ) for each time record, where $U$ is the mean airspeed over the record (used to convert measurements in frequency space to the wave number space).

Similarly, for the temperature structure function parameter $C_{T}^{2}$, the coldwire temperature time series was used, resulting in (Frehlich et al. 2003)

$$
C_{T, \mathrm{UAV}}^{2}=\left(c_{T} / 0.0730846 U^{2 / 3}\right)
$$

(in units of $\mathrm{C}^{2} \mathrm{~m}^{-2 / 3}$ ). Finally, the dissipation rate of temperature variance $\chi_{T}$ is obtained from $\varepsilon$ and $C_{T}^{2}$ via

$$
\chi_{T}=0.9309 C_{T}^{2} \epsilon^{1 / 3}
$$

A procedure similar to that for $C_{T}^{2}$ is used to estimate the refractive index structure function parameter $C_{n}^{2}$, hereafter denoted as $C_{n, \mathrm{UAV}}^{2}$ using the time series of refractive index $n$ :

$$
C_{n, \mathrm{UAV}}^{2}=c_{n} /\left(0.0730846 U^{2 / 3}\right)
$$

For vertical profiles of these estimates, the pressure altitude was averaged over each corresponding time

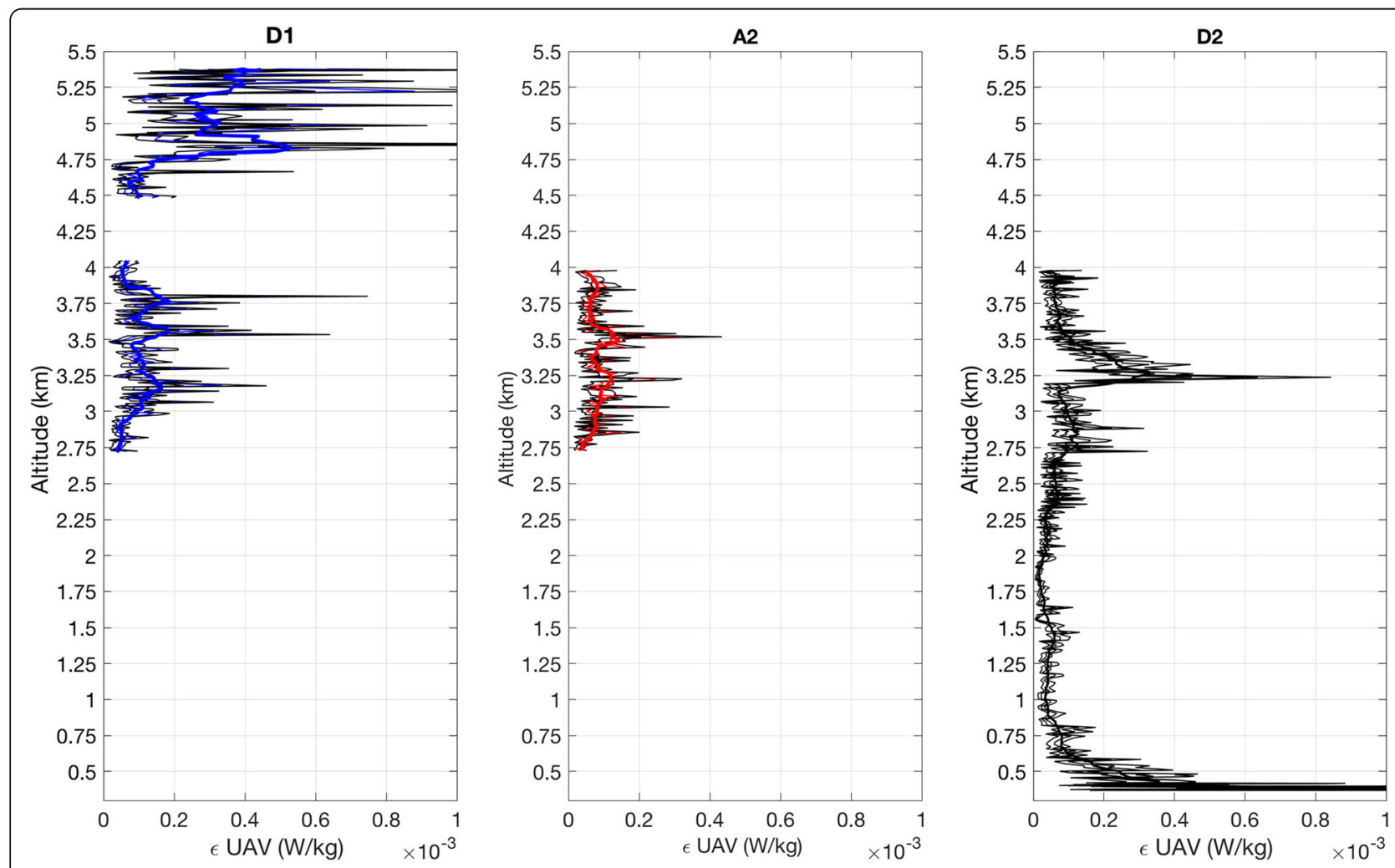

Fig. 16 Vertical profiles of $\varepsilon_{U A V}$ during D1 (left panel), A2 (middle panel), and D2 (right panel) of UAV 17. Thick lines show 11-point (55 s) running means. The thin black lines show the lower and upper bounds on the estimated $\varepsilon_{U A V}$ values 
record. At the nominal ascent/descent rates of $2 \mathrm{~ms}^{-1}$, this provides an altitude resolution of approximately $10 \mathrm{~m}$ in each of these derived quantities.

Derivation of the TKE dissipation rate from velocity spectra followed a similar procedure.

The robustness of the results depends very much on the width of this inertial subrange (flat portion of the weighted spectrum), which should preferably extend at least over one decade in frequency. The coldwire data from this campaign was susceptible to radio frequency interference from the telemetry radio on the UAV, resulting in noise spikes at the $10 \mathrm{~Hz}$ broadcast downlink rate. Future developments will address these extraneous noise sources.

\section{Results}

A total of 19 science flights were made during the ShUREX campaign, between June 5 and June 13, 2015 (see Table 3). The UAV flights managed to sample all four types of structures encountered in the lower troposphere during the campaign, namely, KHI, MCT, convective boundary layer (CBL), and SL. KHI was the hardest, but UAVs 4 to 7 managed to sample weak KHI events occurring at an altitude of $2 \mathrm{~km}$. MCT events tended to last long enough for the UAV to be launched and directed to sample the event. CBL was the easiest to sample, especially when daytime heating produced a deep layer. In all cases, turbulence parameters were derived from the fast response velocity (pitot tube) and temperature (cold wire) sensors on the UAV. Analysis time for all the UAV time series is $5 \mathrm{~s}$, although 11-point (55 s) running means are also computed. The altitudes are above mean sea level (ASL) values, the altitude of the launch site being $358 \mathrm{~m}$.

\section{UAV 5}

Figure 10 shows the radar echo intensity as a function of altitude and time, between 06:00 and 12:00 LT on June 7,2015 . The atmospheric column below $4 \mathrm{~km}$ altitude consists of mostly SL structures, except for a shallow CBL adjacent to the ground and a thin but persistent $\mathrm{KH}$ layer around the altitude of $2.0 \mathrm{~km}$. From left to right, traces of flights UAV 4, 5, 6, and 7 can be seen. We will look at only one of these flights, UAV 5, since the atmospheric structure was very much similar during all four flights. The left panel of Fig. 11 shows the UAV altitude as a function of time, whereas the right panel shows the speed of the UAV relative to the ambient air. Ascent is shown in blue and descent in black. The effect of spiraling ascent and descent can be seen in the relative speed. The relative speed change during a single spiral ranges roughly between 1 and
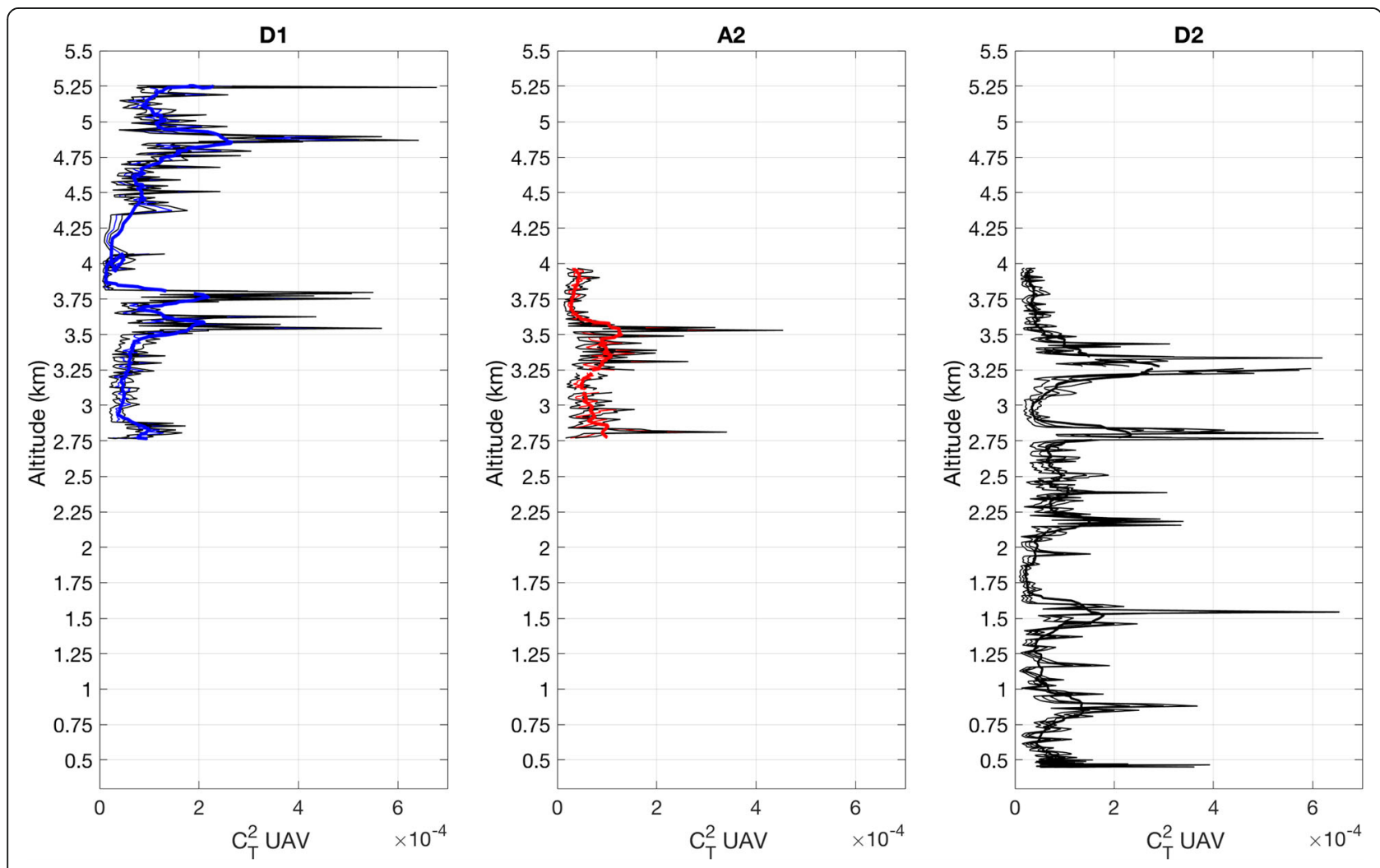

Fig. 17 As in Fig. 16, but for $C_{T, \text { UAV }}^{2}$ 
$3 \mathrm{~ms}^{-1}$, the magnitude of this variation being of course a function of the ambient wind speed at that altitude.

The top panel of Fig. 12 shows $\varepsilon_{\mathrm{UAV}}$ values plotted as a function of time for the same flight (UAV 5). The thin blue line shows the mean values, whereas the thick blue line shows the 11-point running mean. The thin black lines are the upper and lower bounds on the $\varepsilon_{\mathrm{UAV}}$ estimates. The bottom panel shows the UAV altitude as a function of time. This plot should be examined along with Fig. 10, which shows atmospheric structures detected by the radar along with the UAV trace. The peaks in $\varepsilon_{\mathrm{UAV}}$ correlate well with the structures detected by the radar (see Fig. 10). The high noise floor of about $10^{-4} \mathrm{~W} \mathrm{~kg}^{-1}$ in $\varepsilon$ is due to the high noise level of the pitot tube sensor. We intend to replace it in future campaigns with a higher frequency response and lower noise level sensor.

Figure 13 shows vertical profiles of $\varepsilon_{\mathrm{UAV}}$ and $\varepsilon_{\text {radar }}$ during the ascent (left two panels) and descent (right two panels) of UAV 5. The thin red (ascent) and blue (descent) lines indicate the mean $\varepsilon_{\mathrm{UAV}}$ value and the thin black lines show the lower and upper bounds on $\varepsilon_{\mathrm{UAV}}$ estimates (10\% error bounds, i.e., 10\% change in least squares fit error). The thick red (ascent) and blue (descent) lines show the 11-point (55 s) running mean. Apart from the peaks around $2 \mathrm{~km}$ and $1.4 \mathrm{~km}$ altitudes,
$\varepsilon_{\mathrm{UAV}}$ stays around $10^{-4} \mathrm{~W} \mathrm{~kg}^{-1}$, which can be taken as the lower threshold of $\varepsilon_{\mathrm{UAV}}$ measurements. The $\varepsilon_{\text {radar }}$ profiles shown are for altitudes above $1.5 \mathrm{~km}$, the thick red (ascent) and blue (descent) lines showing the mean value and the thin black lines, the upper and lower bounds (i.e., the average and the standard deviations of the values from the mean over $2 \mathrm{~min}$ ). The radar cannot provide reliable data below $1.5 \mathrm{~km}$ altitude in the present case. The dissipation rate enhancements around 1.5 and $2.0 \mathrm{~km}$ are due to turbulence generated by $\mathrm{KHI}$ and by convection at the top of the cloudy $\mathrm{CBL}$, respectively. At these altitudes, the $\varepsilon_{\text {radar }}$ peaks agree well in location, shape, and amplitude with the $\varepsilon_{\mathrm{UAV}}$ peaks.

The $\varepsilon_{\text {radar }}$ and $\varepsilon_{\text {UAV }}$ profiles during descent also show fair agreement between the two. Recall that the launch site is about a kilometer from the radar site and depending on the wind component along the line connecting the two sites, the advection time of turbulent structures could be anywhere from 2 to $10 \mathrm{~min}$.

\section{UAV 17}

The balloon-launched flight UAV 17 managed to sample an MCT event, albeit only in its decaying stages, when turbulence was considerably weaker. Unfortunately, we were not able to launch the UAV in time to sample the initial, highly turbulent phases of the MCT, as can be
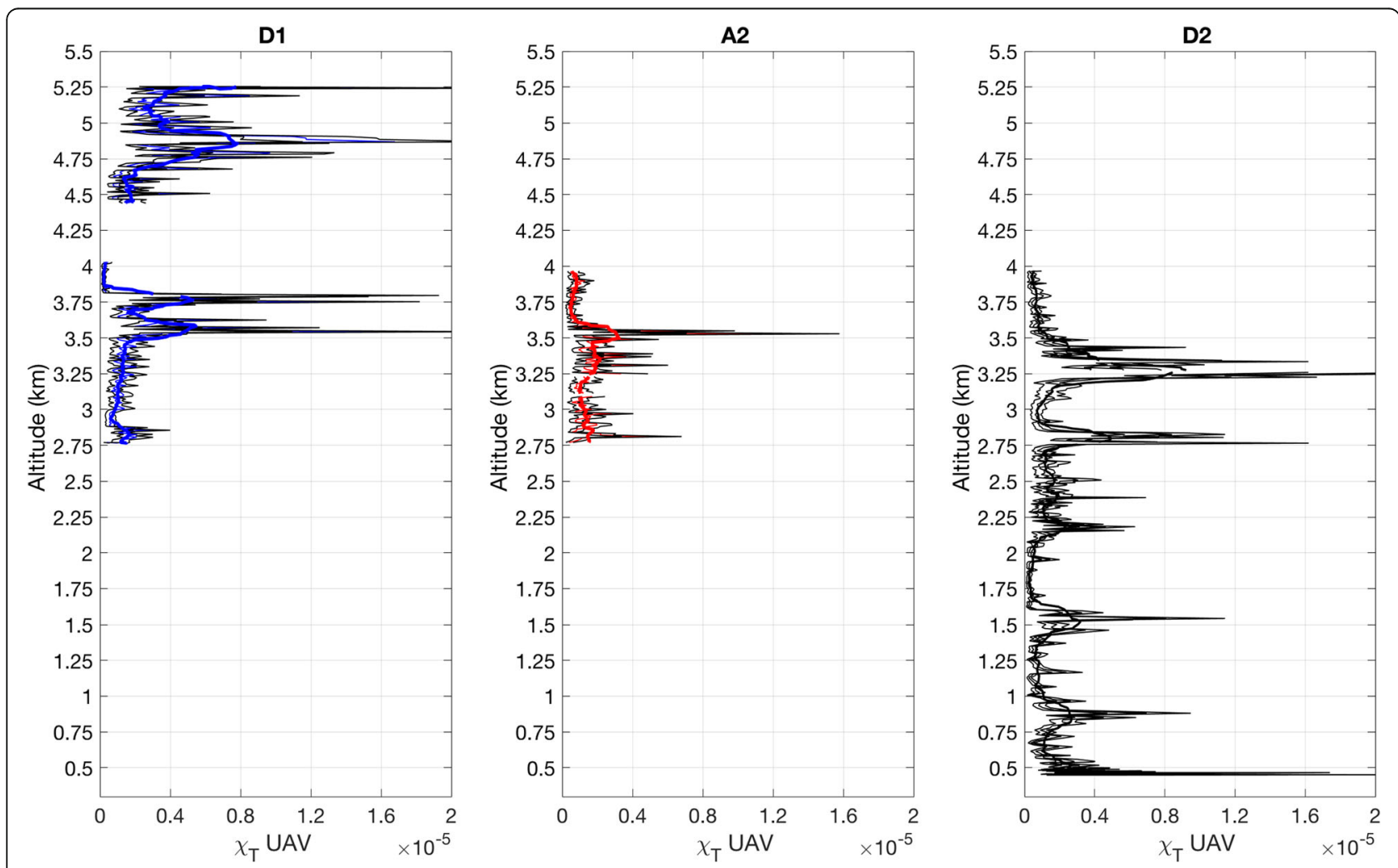

Fig. 18 As in Fig. 16, but for $X_{T, U A V}$ 
seen from Fig. 5a. The left panel of Fig. 14 shows the UAV altitude as a function of time, whereas the right panel shows the speed of the UAV relative to the ambient air. During the initial balloon ascent (denoted as ascent A1) to about $5.35 \mathrm{~km}$, the UAV was attached to the balloon and therefore there is no valid turbulence data from the turbulence sensors, although PTU data are available for this segment. Once the UAV separated from the balloon and started descending (descent D1), turbulence sensors started providing valid data. Once the UAV descended to $2.75 \mathrm{~km}$ altitude, it was commanded to ascend again (ascent A2) to an altitude of $4.0 \mathrm{~km}$, before its final descent (descent D2) to ground level. The relative speed during D1 is shown in blue, A2 in red, and D2 in black. The relative speed change during a single spiral again ranges between roughly 1 and $3 \mathrm{~ms}^{-1}$.

Notice the data gap between 4.5 and $4 \mathrm{~km}$ altitudes during D1. This was due to the fact that the UAV dropped precipitously due to pitot blockage, which prevented the UAV from being flown properly by the flight control software.

The top panel of Fig. 15 shows $\varepsilon_{\mathrm{UAV}}$ (blue) and $C_{T}^{2}$ (red) plotted as a function of time during flight UAV 17. Thin lines show the mean ( $5 \mathrm{~s}$ interval) values, whereas thick lines show the 11-point running mean values. The $C_{T}^{2}$ and $\varepsilon_{\mathrm{UAV}}$ values are comparable in magnitude. The bottom panel shows the UAV altitude as a function of time. This plot should be examined along with Fig. 5b, which shows atmospheric structures detected by the radar, along with the UAV trace. The decaying MCT and $\mathrm{CBL}$ regions can be clearly seen. The highly variable levels of turbulence within these structures can also be seen in this plot. In Fig. 15 and subsequent figures, the unit of $C_{T}^{2}$ is $\mathrm{K}^{2} \mathrm{~m}^{-2 / 3}$. The unit of $\varepsilon$ in all figures is $\mathrm{W} \mathrm{kg}^{-1}$.

Figure 16 shows the vertical profiles of the 11-point running mean of $\varepsilon_{\mathrm{UAV}}$ during D1, A2, and D2 (thick blue, red, and black lines, respectively), whereas the thin blue, red, and black lines (barely visible) show the mean values. The thin black lines in each of the three panels show the upper and lower limits, the difference between the two indicating a measure of the degree of uncertainty in dissipation rate estimates. During D1, the UAV managed to sample a region of high-level turbulence between 5.35 and $4.5 \mathrm{~km}$, where the running mean $\varepsilon_{U A V}$ ranges in value between 0.1 and $0.5 \times 10^{-3} \mathrm{~W} \mathrm{~kg}^{-1}$. This region can be seen clearly in the radar echo in Fig. 5b. At about $4 \mathrm{~km}$ altitude, the UAV encounters the decaying $\mathrm{MCT}$ region, where running mean value ranges between $0.05 \times 10^{-3}$ and $0.2 \times 10^{-3} \mathrm{~W} \mathrm{~kg}^{-1}$. Similar values
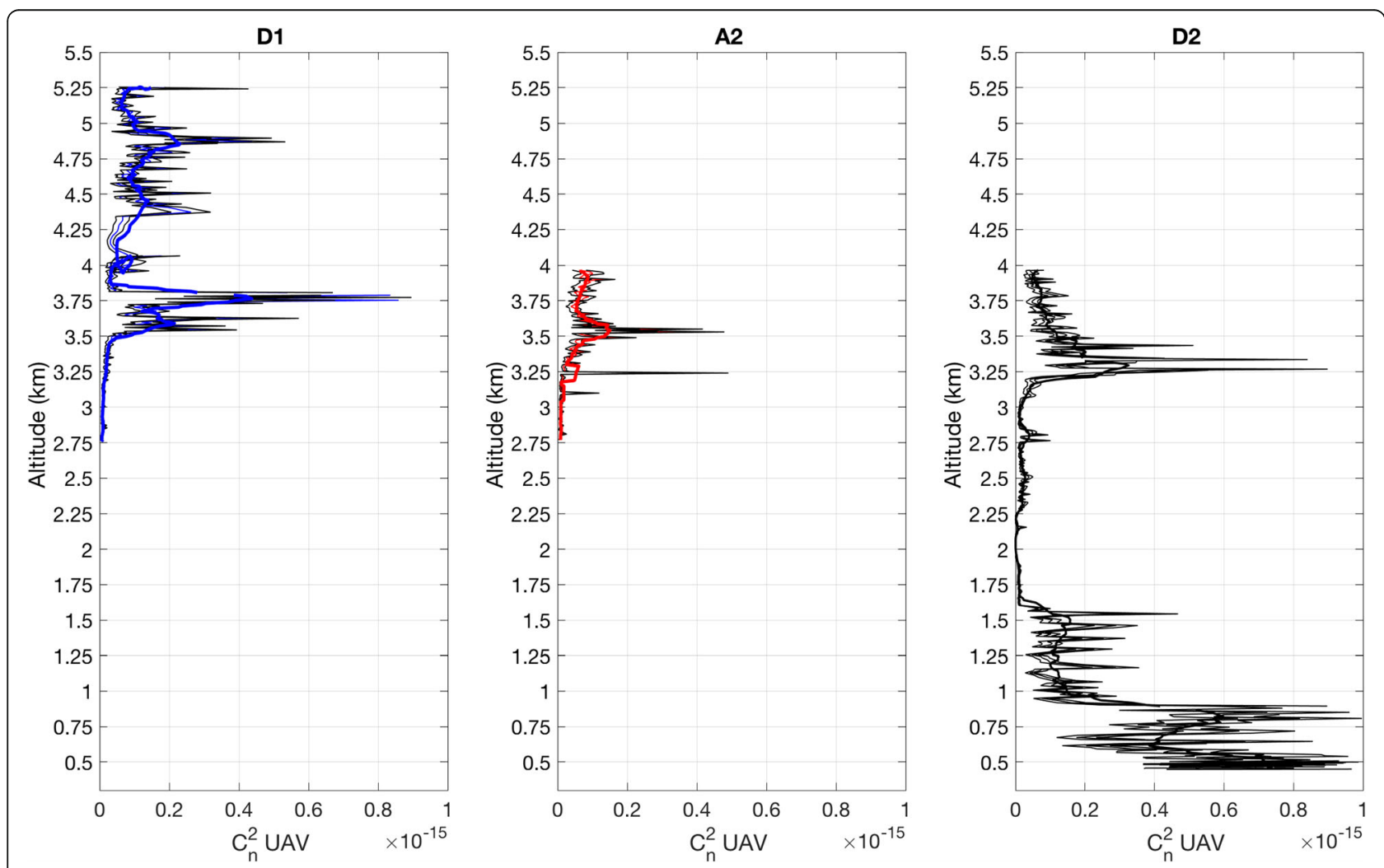

Fig. 19 As in Fig. 16, but for $C_{n, \text { UAV }}^{2}$ 
are measured during A2 through the same region of the MCT. During D2, the UAV encounters a strong turbulent region at altitudes between 3.5 and $3.25 \mathrm{~km}$, where running mean $\varepsilon_{\mathrm{UAV}}$ reaches a maximum of $0.4 \times 10^{-3} \mathrm{~W} \mathrm{~kg}^{-1}$. This region can also be seen clearly in Fig. 5b. During the later part of D2, $\varepsilon_{\mathrm{UAV}}$ hovers close to the threshold value of about $0.02 \times 10^{-3} \mathrm{~W} \mathrm{~kg}^{-1}$ except when the UAV encounters the CBL below $0.75 \mathrm{~km}$ altitude. During the three repeat samplings from 2.7 to $4 \mathrm{~km}$, the locations of the prominent peak moves from 3.6 and $3.8 \mathrm{~km}$ (left panel) to $3.5 \mathrm{~km}$ (middle panel) and then to $3.3 \mathrm{~km}$ (right panel), confirming the descent of the turbulent layer seen in the radar image (Fig. 5b).

Figure 17 shows the corresponding vertical profiles of $C_{T, \mathrm{UAV}}^{2}$ for UAV 17 . The values are comparable in magnitude to $\varepsilon_{\mathrm{UAV}}$. In general, the peaks of $C_{T, \mathrm{UAV}}^{2}$ and $\varepsilon_{\mathrm{UAV}}$ tend to be co-located in altitude, but not always. For example, during D2, several peaks of $C_{T, \mathrm{UAV}}^{2}$ can be seen below $3 \mathrm{~km}$ altitude, whereas similar peaks in $\varepsilon_{\mathrm{UAV}}$ are missing. Figure 18 shows corresponding values of $\chi_{T, \mathrm{UAV}}$ (in $\mathrm{k}^{2} \mathrm{~s}^{-1}$ ) derived from $C_{T, \mathrm{UAV}}^{2}$ and $\varepsilon_{\mathrm{UAV}}$ using Eq. (8).

Figure 19 shows the corresponding vertical profiles of $C_{n, \mathrm{UAV}}^{2}\left(\right.$ in $\mathrm{m}^{-2 / 3}$ ) for UAV 17 . Recall that the lower frequency response of humidity sensor compared to the temperature sensor has some effect on these $C_{n, \mathrm{UAV}}^{2}$ values. Nevertheless, these plots are interesting, since they can be at least qualitatively compared with $C_{n \text {,radar }}^{2}$ profiles.

Figures 20 and 21 show the vertical profiles of $\varepsilon_{\text {radar }}$ and $C_{n, \text { radar }}^{2}\left(\right.$ in $\mathrm{m}^{-2 / 3}$ ), respectively. Recall that the vertical resolution of the radar is much coarser than that of the UAV and so comparisons are best made with the 11point running mean values for the UAV (thick lines in Figs. 16 and 19). Note also that the radar estimates are available only above $1.27 \mathrm{~km}$. In general, there is a good qualitative and quantitative agreement between $\varepsilon_{\text {radar }}$ and $\varepsilon_{\mathrm{UAV}}$. There is also a good qualitative agreement between $C_{n, \text { radar }}^{2}$ and $C_{n, \mathrm{UAV}}^{2}$, but the magnitudes differ significantly.

$C_{T, \mathrm{UAV}}^{2}, C_{n, \mathrm{radar}}^{2}$, and $\chi_{T, \mathrm{UAV}}$ all display peaks at $2.7,2.2$, 1.5 , and at $0.8 \mathrm{~km}$. The $C_{n, \text { radar }}^{2}$ and $\varepsilon_{\text {radar }}$ profiles agree well with the UAV data, with identifiable peaks in the same altitude ranges. The higher layered structures occur in a sharp humidity gradient where warm, humid air descends with time, and the 2.2, 1.5, and $0.8 \mathrm{~km}$ peaks occur at sharp temperature drops as the UAV enters regions of high humidity, due likely to clouds.

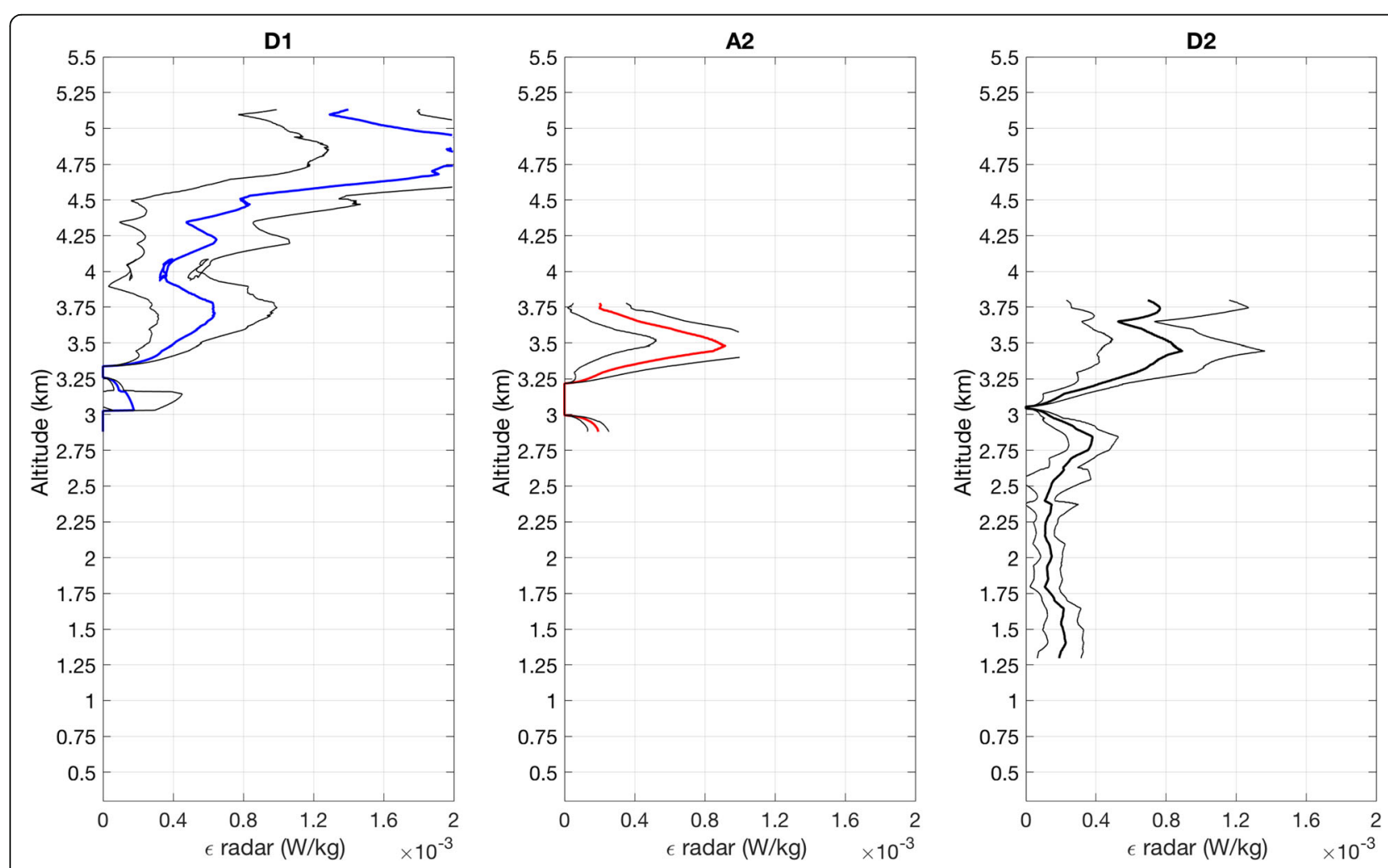

Fig. 20 Vertical profiles of $\varepsilon_{\text {radar }}$ during D1 (left panel), A2 (middle panel), and D2 (right panel) of UAV 17. Thick lines show 11-point (55 s) running means. The thin black lines show the lower and upper bounds on the estimated $\varepsilon_{\text {radar }}$ values 

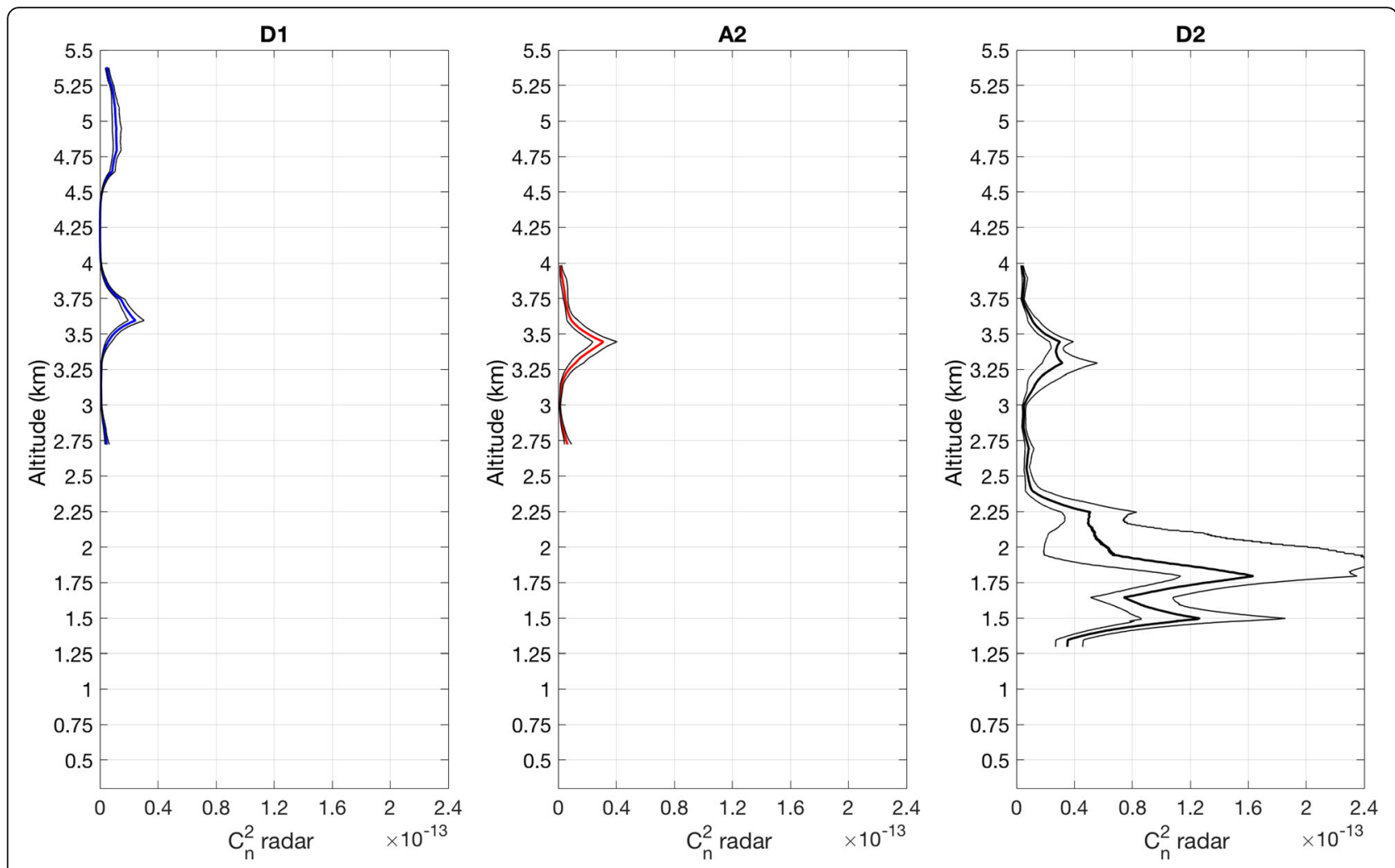

Fig. 21 As in Fig. 20, but for $C_{n, \text { radar }}^{2}$

\section{Vaisala radiosondes}

An example of the radiosonde and UAV flight tracks superimposed on the image of radar echo power at vertical incidence is shown in Fig. 22. The Vaisala sonde was released 15 min after the launch of the UAV 4 on June 7,2015 , and ascended at roughly the same speed $\left(2 \mathrm{~ms}^{-1}\right)$ as the UAV. Figure 23 shows the vertical profiles of temperature and humidity from the UAV-borne iMET and Vaisala sondes. Below the height of $1.4 \mathrm{~km}$, both Vaisala and iMET sondes indicate air saturation with respect to liquid water, even though $\mathrm{RH}$ of $100 \%$ for the Vaisala sonde likely due to differences in calibration (also detectable all along the profiles). Between the heights of $\sim 1.4 \mathrm{~km}$ and $\sim 2.5 \mathrm{~km}$, the temperature and relative humidity profiles measured by the iMET and Vaisala sondes show significant differences despite the proximity of the

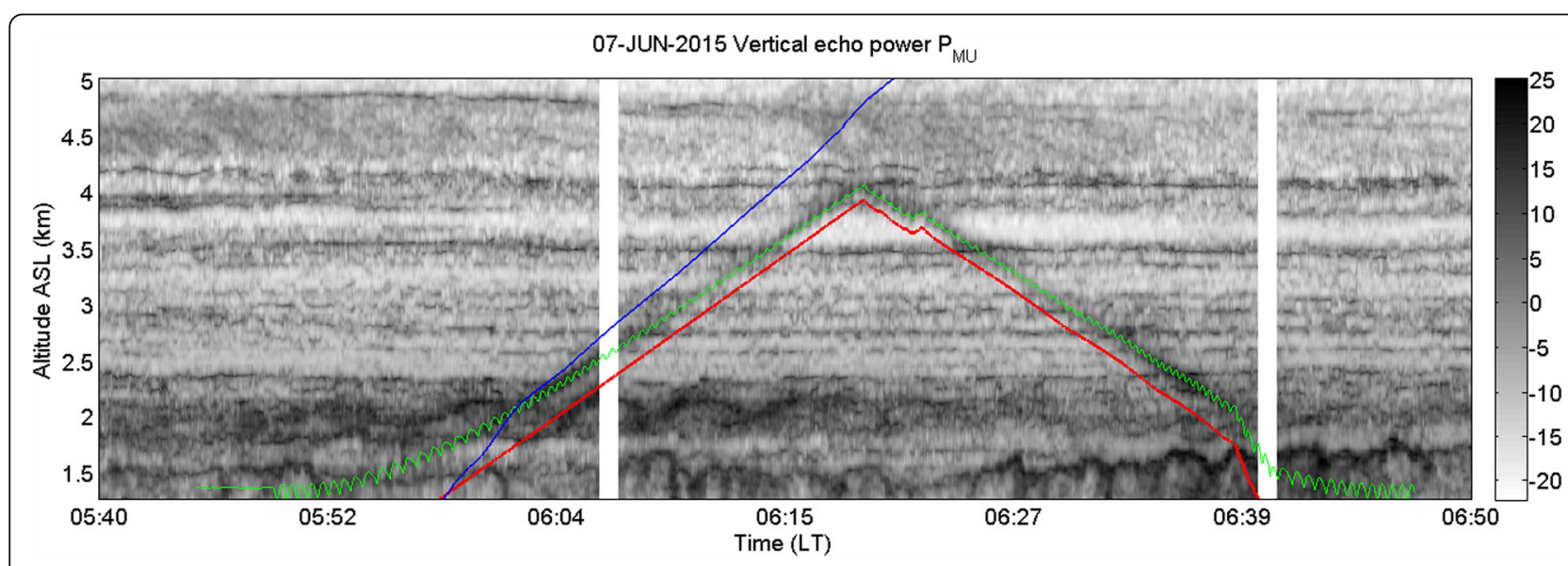

Fig. 22 Close-up of Fig. 10 around the time period of flight UAV 4 showing its altitude (red line) and the altitude of the Vaisala sonde (blue line) released on June 7,2015 . The green curve shows the range to the UAV with respect to the center of the MU radar antenna 

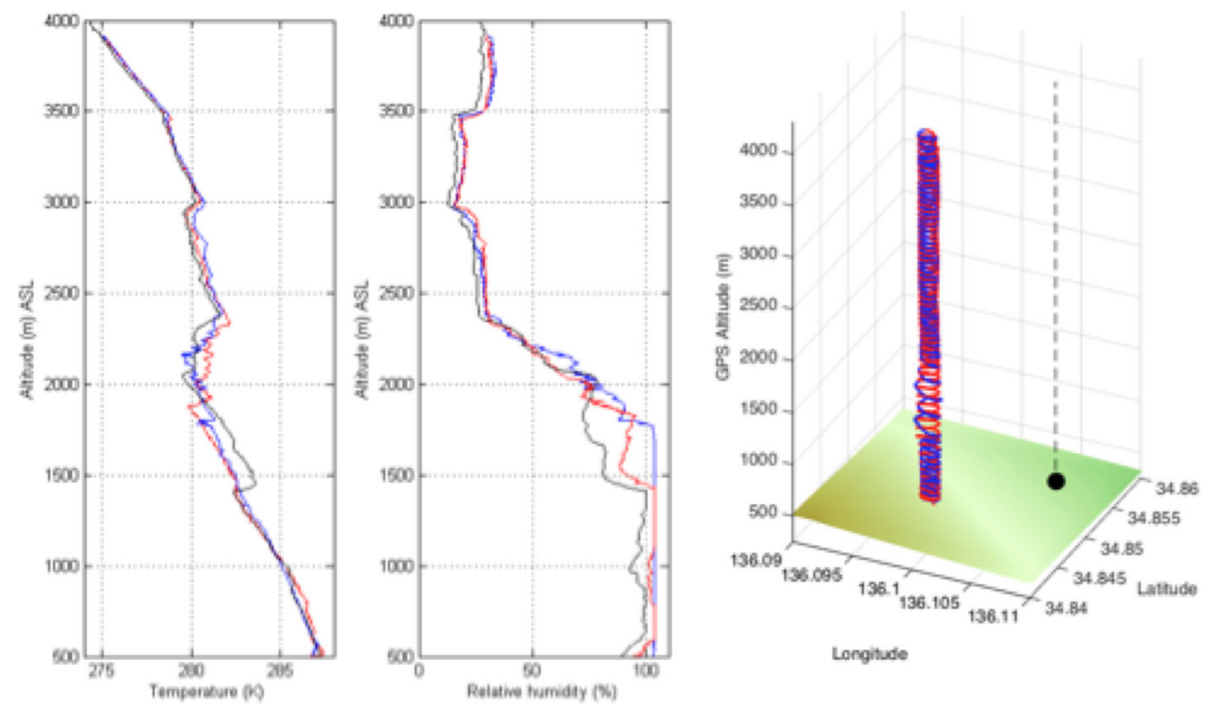

Fig. 23 (Left panel) Vertical profiles of temperature and relative humidity from the Vaisala sonde (black curves) and the UAV-borne iMET sonde (red curves ascent, blue curves descent) for UAV 4. (Right panel) The trajectory of UAV 4 (red ascent, blue descent). The Vaisala sonde was launched from the MU radar site (indicated by the black circle) 15 min after the UAV was launched and ascended at a nominal rate of about $2 \mathrm{~ms}^{-1}$

measurements in time and space. These features contrast with the remarkable resemblance of the iMET and Vaisala profiles above the height of $2.4 \mathrm{~km}$. These datasets are analyzed in detail and compared with radar data by (Luce $\mathrm{H}$, Hashiguchi $\mathrm{H}$, Kantha L, Lawrence D, Tsuda T, Mixa T: Concurrent MU radar, UAV and balloon observations of temperature and moisture fine-scale structures during the ShUREX 2015 campaign, in preparation).

\section{Conclusions}

The ShUREX 2015 campaign is the first time a UAV has been used extensively to probe turbulent structures in the lower atmosphere, guided in real or near real time by measurements from a VHF radar operated concurrently in high vertical resolution range imaging mode. The goal of the campaign was to measure turbulence parameters in the lower atmosphere, along with concurrent radar-derived values. While Scipion et al. (2016) made simultaneous observations of structure function parameter of refractive index using DataHawk and the Jicamarca radar, reliable and accurate measurements of a suite of turbulence parameters, including the TKE dissipation rate, were possible only during the ShUREX campaign, because of the extensive effort needed to improve the turbulence sensors and processing of turbulence data.

The campaign was quite successful in achieving the goals set forth at the outset. It unambiguously demonstrated the utility of a small, light weight, inexpensive, autonomous UAV, such as DataHawk, in probing the lower atmosphere and of the synergistic use of a VHF radar and UAVs. We were able to sample interesting atmospheric structures such as sheets and layers and MCT, guided in real time or near real time by the radar images. Salient results have been obtained (as illustrated in this paper) and are described in greater detail in related publications (Luce $\mathrm{H}$, Hashiguchi $\mathrm{H}$, Kantha $\mathrm{L}$, Lawrence D, Tsuda T, Mixa T: On the performance of the range imaging technique using UAVs during the ShUREX 2015 campaign, submitted; Luce et al. 2017; Luce H, Hashiguchi H, Kantha L, Lawrence D, Tsuda T, Mixa T: Concurrent MU radar, UAV and balloon observations of temperature and moisture fine-scale structures during the ShUREX 2015 campaign, in preparation). Turbulence parameters, such as the TKE dissipation rate $\varepsilon$ and the refractive index structure function parameter $C_{n}^{2}$ inferred from radar signals, are consistent with in situ measurements by sensors flown on the UAV in the vicinity of the radar.

The campaign also demonstrated the validity and utility of the range imaging technique in obtaining very high vertical resolution $(\sim 20 \mathrm{~m})$ images of the atmospheric column, which display fine-scale atmospheric structures in unprecedented detail. These images help us better understand the generation, evolution, and dissipation of such structures and associated dynamical processes.

However, the less-than-optimal frequency response $(100 \mathrm{~Hz})$, combined with the high noise level of the cold wire and pitot sensors, prevented the use of the spectra above a certain frequency, leading to rather narrow inertial subranges in the turbulence spectra. Higher 
frequency response sensors with much lower noise floor should yield broader inertial subranges and hence enable more accurate derivation of the TKE dissipation rate $\varepsilon$ and turbulence structure parameters such as $C_{T}^{2}$ and $C_{n}^{2}$.

In addition, the vibrations induced by the motor contaminated the turbulence spectra during ascent (and occasionally during descent, when the throttle was high) and the discrete frequency spikes in the data had to be removed before deducing $\varepsilon, C_{T}^{2}$, and $C_{n}^{2}$ from UAV data. It would be far better to have spectra not contaminated by motor vibrations in the inertial subrange, which is the goal of future ShUREX campaigns.

One problem we had during ShUREX was the inability to fly DataHawk in winds above $10 \mathrm{~ms}^{-1}$ due to a suboptimum flight control algorithm for upwind segments. Partly because of strong winds aloft, we were unable to sample highly interesting structures above $5 \mathrm{~km}$. Communication dropout caused losses of two UAVs during the campaign. These deficiencies have been corrected, and during future ShUREX campaigns, DataHawk can fly to altitudes of up to $7.0 \mathrm{~km}$ (if permitted by the aviation authorities) and in stronger winds (as much as $18 \mathrm{~ms}^{-1}$ ), once again guided by real-time or near realtime radar signals. A higher capacity LiPo battery pack can also increase flight times to around $2 \mathrm{~h}$.

Since relative velocity measurements made by a pitot tube are strongly affected by any structural vibrations induced by the motor and the propeller, the TKE dissipation rates derived require careful interpretation in conjunction with the throttle level. During future ShUREX campaigns, this problem can be alleviated by turning off the motor and simply gliding down through the atmospheric feature of interest, after a climb to a pre-determined altitude. This has been made possible by a more robust flight control algorithm. Better yet, the DataHawk can also be equipped with a hot wire sensor, and lower noise level cold wire and pitot tube. The data collected should therefore enable even more accurate measurements and a better understanding of turbulent processes in the troposphere, including KHI-induced turbulence and MCT. We will continue to make turbulence measurements at the Shigaraki MU radar site in the future, but with improved sensors and DataHawk capabilities. The technical and scientific outcomes will be disseminated in a timely fashion through professional meetings such as JpGU/AGU and MST radar conferences, and journal publications.

A major limitation on UAV probing of atmospheric structures is the constraint imposed by aviation authorities. This includes the limit on altitude and prohibition of balloon launches and nocturnal flights. This means the upper troposphere and nighttime atmosphere cannot be probed.

\section{Abbreviations}

ABL: Atmospheric boundary layer; CBL: Convective boundary layer; CW: Cold wire; DH: DataHawk; GPS: Global positioning system; iMET: International Met Systems; KHI: Kelvin-Helmholtz instability; MCT: Mid-level cloud-base turbulence; MU: Middle and Upper Atmosphere; PTU: Pressure, temperature, humidity; RH: Relative humidity; RISH: Research Institute for Sustainable Humanosphere; ShUREX: Shigaraki UAV-Radar Experiment; SL: Sheets and layers; TKE: Turbulent kinetic energy; UHF: Ultra high frequency;

UAV: Unmanned aerial vehicle; VHF: Very high frequency

\section{Acknowledgements}

LK, DL, and TM thank the Japanese Society for Promotion of Science (JSPS) and RISH for providing the funding to participate in the campaign as well as to modify the DataHawk to carry the iMET sonde. The stays of HL and RW were funded by CNRS PICS. The hospitality of RISH personnel and director Prof. Tsuda to their visitors is exemplary. Thanks to Abhiram Doddi for the help with the analysis of UAV data.

\section{Funding}

This work was supported by JSPS KAKENHI Grant Number JP15K13568. The $\mathrm{MU}$ radar belongs to and is operated by the Research Institute for Sustainable Humanosphere (RISH), Kyoto University.

\section{Authors' contributions}

LK conceived and led the campaign and participated in the analysis and synthesis of the study results. DL flew the UAV and collected and processed the turbulence data. $\mathrm{HL}$ collected and analyzed the MU radar data with the assistance from $\mathrm{HH}, \mathrm{T}$, and MY. TM helped fly the UAV and participated in the data analysis. RW collected and analyzed the radiosonde data. All authors have read and approved the final manuscript.

\section{Authors' information}

TT was the Director of RISH, Kyoto University, which operates the MU radar, and was assisted in this task by $\mathrm{HH}$ and MY. LK and DL are professors in the Department of Aerospace Engineering Sciences at the University of Colorado,

Boulder. TM is a doctoral student of LK. RW is an associate professor at Université Pierre et Marie Curie, Paris, France, and HL is an associate professor at Université de Toulon, France.

\section{Competing interests}

The authors declare that they have no competing interests.

\section{Publisher's Note}

Springer Nature remains neutral with regard to jurisdictional claims in published maps and institutional affiliations.

\section{Author details}

${ }^{1}$ Aerospace Engineering Sciences, University of Colorado, Boulder, CO 80309, USA. ${ }^{2}$ Mediterranean Institute of Oceanography (MIO), Université de Toulon, CNRS/INSU, UM 110, Toulon, France. ${ }^{3}$ Research Institute for Sustainable Humanosphere, Kyoto University, Kyoto, Japan. ${ }^{4}$ LATMOS-IPSL, Université Pierre et Marie Curie (UPMC), CNRS-INSU, Paris, France.

Received: 24 January 2017 Accepted: 8 June 2017

Published: 17 July 2017

\section{References}

Balsley BB, Lawrence DA, Woodman RF, Fritts DC (2013) Fine-scale characteristics of temperature, wind, and turbulence in the lower atmosphere $(0-1,1300 \mathrm{~m})$ over the south Peruvian coast. Boundary Layer Meteor 147:165-178. doi:10. 1007/s10546-012-9774-x

Bonin TA, Goines DC, Scott AK, Wainwright CE, Gibbs JA, Chilson PB (2015) Measurements of temperature structure-function parameters with a small unmanned aerial system compared with a sodar. Boundary Layer Meteor 155:417-434. doi:10.1007/s.10546-015-0009-9

Doviak RJ, Zrnic' DS (1984) Reflection and scatter formula for anisotropically turbulent air. Radio Sci 19:325-336

Frehlich R, Meillier Y, Jensen M, Balsley B (2003) Turbulence measurements with the CIRES TLS (tethered lifting system) during CASES-99. J Atmos Sci 60: $2487-2495$ 
Fukao S, Hamazu K (2014) Radar Observations of the Clear Atmosphere, Radar for meteorological and atmospheric observations. Springer, Tokyo, pp 223-267. doi:10.1007/978-4-431-54334-3_7

Fukao S, Sato T, Tsuda T, Yamamoto M, Yamanaka MD (1990) MU radar-new capabilities and system calibrations. Radio Sci 25:477-485

Fukao S, Yamanaka MD, Ao N, Hocking WK, Sato T, Yamamoto M, Nakamura T, Tsuda T, Kato S (1994) Seasonal variability of vertical eddy diffusivity in the middle atmosphere. 1. Three-year observations by the middle and upper atmosphere radar. J Geophys Res Atmos 99:18973-18987

Gossard EE, Strauch RG (1983) Radar observations of clear air and clouds. Elsevier, New York, p 280

Hocking WK (1983) On the extraction of atmospheric turbulence parameters from radar backscatter Doppler spectra-I. Theory. J Atmos Terr Phys 45:89-102

Hocking WK (1985) Measurement of turbulent energy dissipation rates in the middle atmosphere by radar techniques. Radio Sci 20:1403-1422

Hocking WK (1999) The dynamical parameters of turbulence theory as they apply to middle atmosphere studies. Earth Planet Space 51:525-541

Hocking WK (2011) A review of mesosphere-stratosphere-troposphere (MST) radar developments and studies, circa 1997-2008. J Atmos Sol Terr Phys 73:848-882

Holton JR (2004) Introduction to dynamic meteorology, 4th edn. Elsevier, New York

Hooper DA, Arvelius J, Stebel K (2004) Retrieval of atmospheric stability from MST radar return signal power. Ann Geophys 22:3781-3788

Kato S (2005) Review: middle atmosphere research and radar observation. Proc Japan Acad 81, nb8, pp. 306-320

Kudo A (2013) The generation of turbulence below midlevel cloud bases: the effect of cooling due to sublimation of snow. J Appl Meteorol Climatol 52: 819-833

Kudo A, Luce H, Hashiguchi H, Wilson R (2015) Convective instability underneath midlevel clouds: comparisons between numerical simulations and VHF radar observations. J Appl Meteorol Climatol 54:2217-2227

Lawrence DA, Balsley BB (2013) High-resolution atmospheric sensing of multiple atmospheric variables using the DataHawk small airborne measurement system. J Atmos Ocean Technol 30:2352-2366

Lawrence DA, Frew E, Pisano W (2008) Lyapunov vector fields for autonomous UAV flight control. AIAA J Guid Control Dyn 31:1220-1229

Luce H, Yamamoto M, Fukao S, Hélal D, Crochet M (2001) A frequency radar interferometric imaging applied with high resolution methods. J Atmos Sol Terr Phys 63:221-234

Luce H, Hassenpflug G, Yamamoto M, Fukao S (2007) Comparisons of refractive index gradient and stability profiles measured by balloons and the MU radar at a high vertical resolution in the lower stratosphere. Ann Geophys 25:47-57

Luce H, Wilson R, Dalaudier F, Hashiguchi H, Nishi N, Shibagaki Y, Nakajo T (2014) Simultaneous observations of tropospheric turbulence from radiosondes using Thorpe analysis and the VHF MU radar. Radio Sci 49:1106-1123. doi:10. 1002/2013RS005355

Luce H, Hashiguchi H, Kantha L, Lawrence D, Tsuda T, Mixa T, Yabuki M (2017) Comparisons between high-resolution profiles of squared refractive index gradient $\mathrm{M}^{2}$ measured by the MU radar and UAVs during the ShUREX 2015 campaign. Ann Geophys (accepted)

Ottersten H (1969) Atmospheric structure and radar backscattering in clear air. Radio Sci 4:1179-1193

Palmer RD, Yu T-Y, Chilson PB (1999) Range imaging using frequency diversity. Radio Sci 34:1485-1496

Röttger J, Larsen MF (1990) UHFNHF radar techniques for atmospheric research and wind profiler applications. Radar Meteorol Chap 21, Atlas D (ed) 235-281.

Scipion DE, Lawrence DA, Milla MA, Woodman RF, Lume DA, Balsley BB (2016) Simultaneous observations of structure function parameter of reftractive index using a high resolution radar and the DataHawk small airborne measurement system. Ann Geophys (in print).

Tsuda T (2014) Review. Characteristics of atmospheric gravity waves observed using the MU (middle and upper atmosphere) radar and GPS (Global Positioning System) radio occultation. Proc Japan Acad Ser B 90:12-27. doi:10.2183/pjab.90.12

van den Kroonenberg A, Martin T, Buschmann M, Bange J, Vorsmann P (2008) Measuring the wind vector using the autonomous mini aerial vehicle M2AV. J Atmos Ocean Technol 25:1969-1982

White AB, Lataitis RJ, Lawrence RS (1999) Space and time filtering of remotely sensed velocity turbulence. J Atmos Ocean Technol 16(D6):1967-1972

Wilson R, Luce H, Hashiguchi H, Nishi N, Yabuki Y (2014) Energetics of persistent turbulent layers underneath mid-level clouds estimated from concurrent radar and radiosonde data. J Atmos Sol Terr Phys 118:78-89

\section{Submit your manuscript to a SpringerOpen ${ }^{\circ}$ journal and benefit from:}

- Convenient online submission

- Rigorous peer review

- Open access: articles freely available online

- High visibility within the field

- Retaining the copyright to your article

Submit your next manuscript at $\boldsymbol{\nabla}$ springeropen.com 